\title{
Combining operational models and data into a dynamic vessel risk assessment tool for coastal regions
}

\author{
R. Fernandes ${ }^{1, a}$, F. Braunschweig ${ }^{2}$, F. Lourenço ${ }^{2, b}$, and R. Neves ${ }^{1}$ \\ ${ }^{1}$ MARETEC - Marine Environment and Technology Centre, Instituto Superior Técnico, \\ Universidade de Lisboa, Avenida Rovisco Pais, 1049-001, Lisbon, Portugal \\ ${ }^{2}$ Action Modulers, Estrada Principal, 29, 2640-583, Mafra, Portugal \\ anow at: Action Modulers, Estrada Principal, 29, 2640-583, Mafra, Portugal \\ b now at: Aubay Portugal, Av. República, $101-3^{\circ}$ E, 1050-190 Lisbon, Portugal \\ Correspondence to: R. Fernandes (rodrigo.fernandes@actionmodulers.com)
}

Received: 25 May 2015 - Published in Ocean Sci. Discuss.: 9 July 2015

Revised: 4 December 2015 - Accepted: 26 January 2016 - Published: 23 February 2016

\begin{abstract}
The technological evolution in terms of computational capacity, data acquisition systems, numerical modelling and operational oceanography is supplying opportunities for designing and building holistic approaches and complex tools for newer and more efficient management (planning, prevention and response) of coastal water pollution risk events.
\end{abstract}

A combined methodology to dynamically estimate time and space variable individual vessel accident risk levels and shoreline contamination risk from ships has been developed, integrating numerical metocean forecasts and oil spill simulations with vessel tracking automatic identification systems (AIS). The risk rating combines the likelihood of an oil spill occurring from a vessel navigating in a study area - the Portuguese continental shelf - with the assessed consequences to the shoreline. The spill likelihood is based on dynamic marine weather conditions and statistical information from previous accidents. The shoreline consequences reflect the virtual spilled oil amount reaching shoreline and its environmental and socio-economic vulnerabilities. The oil reaching shoreline is quantified with an oil spill fate and behaviour model running multiple virtual spills from vessels along time, or as an alternative, a correction factor based on vessel distance from coast. Shoreline risks can be computed in real time or from previously obtained data.

Results show the ability of the proposed methodology to estimate the risk properly sensitive to dynamic metocean conditions and to oil transport behaviour. The integration of meteo-oceanic + oil spill models with coastal vulnerability and AIS data in the quantification of risk enhances the maritime situational awareness and the decision support model, providing a more realistic approach in the assessment of shoreline impacts. The risk assessment from historical data can help finding typical risk patterns ("hot spots") or developing sensitivity analysis to specific conditions, whereas real-time risk levels can be used in the prioritization of individual ships, geographical areas, strategic tug positioning and implementation of dynamic risk-based vessel traffic monitoring.

\section{Introduction}

Maritime surveillance systems are becoming more effective and have been developed for coastal regions (e.g. terrestrial and satellite-based Automatic Identification Systems - AISs, UAVs), and the maritime security rules are becoming more restrictive, following the MARPOL convention (e.g. shift to ships with double hull). However, the increasing global ship traffic (four times as many ships now as in 1992; Tournadre, 2014) and maritime transport of oil products (ITOPF, 2015) make it more difficult to significantly reduce the environmental, economic and social risks posed by potential spills. Additionally, the use of increasingly larger vessels (up to 100000 150000 tonnes) means that if a major accident takes place, the amount of oil released could be vast.

In fact, the environmental and socio-economic issues associated with spills is and will always be a main topic: spill 
events are continuously happening, most of them unknown to the general public because of their small-scale impact - for instance, half of the total oil spills in the marine environment come from operative discharges by shipping and in most of these cases the discharges are illegal (GESAMP, 2007). Nevertheless, some oil spills become authentic media phenomena in this information era, due to their large dimensions and environmental and social-economic impacts on ecosystems and local communities, and also due to some spectacular or shocking pictures generated (Leschine, 2002).

Consequently, the planning and prevention in the management of spill incidents at sea is extremely important in the reduction and minimization of potential impacts. Latest scientific and technological developments on coastal monitoring and operational oceanography have provided the opportunity to build more complex and integrated decision support systems for coastal risk management. The increasing operational predictive capacity of marine weather conditions (Hurlburt et al., 2009; Schiller, 2011) and better knowledge of the fate and behaviour processes of pollutants spilt at sea or coastal zones (Fingas, 2015; Johansen et al., 2015; Zhao et al., 2014a, b; Gong et al., 2014), together with the presence of advanced surveillance monitoring tools (Fischer and Bauer, 2010), can be integrated in order to provide a safer support for decision making in emergency or planning issues associated with pollution risks.

The development of risk assessment studies have been used for multiple purposes, including contingency planning for response and preparedness, developing spill prevention measures, or evaluating oil exploration sites (Etkin, 2014).

Over the years, innovative oil spill hazard or risk assessment studies in coastal and marine environments have been published, considering historical data, reference situations, and typical or extreme scenarios (Castanedo et al., 2009; den Boer et al., 2014; Otero et al., 2014; WSP Canada Inc., 2014; Liubartseva et al., 2015), supporting contingency planning and strategic decision making. Silveira et al. (2013) developed also a new method to calculate the ship risk collision, applied in the Portuguese continental shelf with AIS data, but without connection to oil spill hazard assessment or taking into consideration metocean conditions. But none of the previous studies were developed and applied in real-time risk assessment.

Other studies and methodologies developed dynamic approaches, with the possibility of being used in real-time support (Grifoll et al., 2010; Eide et al., 2007a, b; Bi and Si, 2012; Olita et al., 2012; Goldman et al., 2015; Canu et al., 2015). However, the method proposed by Grifoll et al. (2010) does not include a fate and behaviour oil spill model for a better determination of areas affected by oil. The work developed by Eide et al. $(2007 \mathrm{a}, \mathrm{b})$ included an oil spill model - however, the simulations were previously obtained, based on typical scenarios, and without considering the dynamic changing of environmental conditions. Bi and $\mathrm{Si}$ (2012) also presented a novel method for dynamic risk assessment of oil spill accidents based on numerical simulation, but in this case the method was only applied to an on-demand spill event or scenario, instead of providing continuous risk mapping based on ship traffic. Olita et al. (2012), Canu et al. (2015) and Goldman et al. (2015) do not integrate directly metocean modelling data in the risk (accident probability) model, and the latter two papers do not use vessel data.

In this work, we present an innovative and holistic methodology for dynamic shoreline risk quantification, with full integration of numerical metocean forecasts and oil spill simulations with the existing monitoring tools (AIS), and with the possibility of being used to study past periods, projected scenarios and also to support continuous monitoring, contributing to real-time maritime situational awareness. The main purpose is to build a decision support system capable of quantifying time and space variable shoreline pollution risk levels, coming from ships along the coast, and combining multiple information layers:

a. instant vessel information (AIS);

b. regional statistics information on vessels accidents history, coastal vulnerabilities;

c. instant metocean forecasting data;

d. continuously simulated oil spill fate and behaviour from ships along the coast.

The development of a risk assessment approach integrating economic, environmental and social aspects combined with operational oceanography and available surveillance monitoring systems is in line with the blue growth paradigm, resulting in an innovative, holistic and sustainable approach for the maritime sector.

The relevance of integrating the oil spill model and metocean data from forecasting systems in the risk algorithm is evaluated on a study area described in the next section.

\section{Materials and methods}

\subsection{Pilot area}

The whole system has been implemented and tested in the Portuguese continental shelf. This peripheral area is a high shipping density zone (more than 55000 commercial vessels per year crossing this area, and an average number of 600 ships of all types present in the studied area, according to MarineTraffic, http://www.marinetraffic.com/) with a complex network of routes, being an obligatory passage point between the Mediterranean Sea and Northern Europe or the American Continent (Silveira et al., 2013; see Fig. 1).

In this geographical zone, the activities in the near-shore area assume a very relevant role in the social, environmental and economic context (vast potential in natural resources, fishing, aquaculture, maritime commerce and port activity, leisure, sports and tourism activities). 


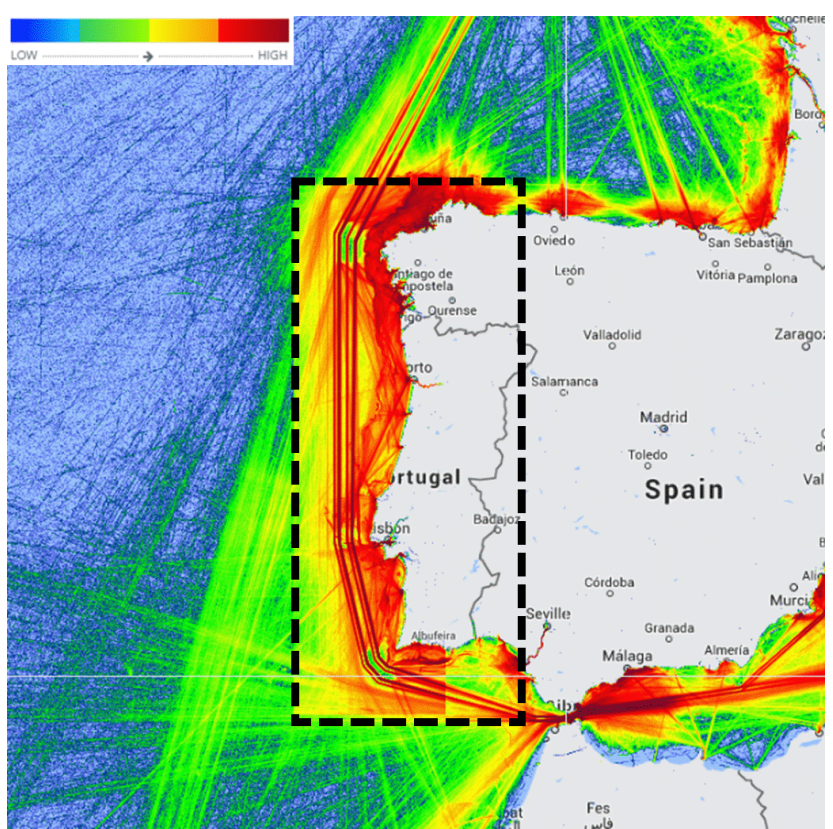

Figure 1. Ship density map around the pilot area in 2014. The white rectangle represents the area considered in this work to study the shoreline contamination risk in the Portuguese continental coast (source: www.marinetraffic.com).

In Portugal, the direct contribution of the maritime economy amounted to about $2.5 \%$ of national gross value added in 2010 and $2.3 \%$ of national employment (DGPM, 2012). Tourism, on the other hand, is gaining an important weight in the economy and is currently representing $48 \%$ of the total employment related to maritime activities (DGPM, 2012), as the country is widely known as a sun and beach destination within Europe counting with a wide accommodation and restoration infrastructure.

The high frequency of ships navigating in the Portuguese coast, together with the Portuguese dependency on the economy of the sea and natural resources, raise the awareness for the risk of water pollution events in this area.

\subsection{Approach}

The method proposed for quantification of risk combines the likelihood of an oil spill occurring from a vessel navigating in the study area with the assessed consequences to the shoreline, where risk is the product of the probability (or frequency) of oil spill accidents from maritime traffic, times the severity (or consequences) of the events:

Risk $=$ Probability $\times$ Severity.

Governed by the previous expression, different types or risk levels are determined:

- the individual risk of oil spill accident for each vessel, depending on the vessel itself and on the metocean con- ditions, which is not dependent on the coastal consequences;

- the risk of shoreline contamination taking in account coastal vulnerability indices with the integration of the above risks of oil spill accidents of all the vessels present in the vicinity of a given coastal stretch. To account for the potential consequences and amount of oil reaching shoreline, two strategies are implemented and evaluated:

- a modelled one using an oil spill transport and behaviour spill modelling for each vessel

- a non-modelled one based on a correction factor function of the distance between the vessel and the coast stretch.

The methodology and some of the statistic data is based on the risk assessment produced for Portugal and Galicia in the scope of the EROCIPS project (Filipe and Pratas, 2007). A previous description of the risk model is available in ARCOPOL plus report (Fernandes, 2014).

The probability is based on dynamic marine weather conditions and statistical information (frequency constants for each accident type) from previous accidents. The severity of the consequences are the result of the combination of hypothetical spilled oil amount reaching shoreline and the coastal vulnerability on those affected areas.

In order to simplify the development of the scale of risk and its values, logarithmic values are used, defined by indices, following IMO recommendations (IMO, 2002):

$\log ($ Risk $)=\log ($ Probability $) \times \log ($ Severity $)$

or

$I_{\mathrm{RSI}}=I_{\mathrm{PSI}}+I_{\mathrm{SSI}}$

Full details of the risk assessment model implemented are given in Sect. 2.7.

\subsection{Vessel information}

Variable vessel information is used in the computation of risk. The properties used are the geographical position, cargo type, speed, vessel type, weight (DWT), name and ID (MMSI and IMO number). Vessels with less than 100 DWT, passenger vessels and fishing vessels navigating outside restricted waters are not considered in this study, based on the assumptions from Filipe and Pratas (2007), and also for computational reasons (the risk model takes in consideration approximately 150 vessels every instant, after applying the mentioned filtering). It is assumed that a vessel is navigating in restricted waters if distance to shoreline is not greater than 3 nautical miles, or if water depth is not deeper than $20 \mathrm{~m}$. 
The vessel information is obtained from AIS data. Presently the system is configured to seamlessly collect realtime data from www.AISHUB.net or MarineTraffic API service, but the system can be easily adapted to collect information from any other online AIS data provider. The system is also prepared to import historical data.

\subsection{Coastal vulnerability}

The coastal vulnerability is used to quantify the consequences of shoreline contamination, with a risk algorithm. This coastal vulnerability can be obtained from different vulnerability indices: the coastal sensitivity index (CSI), the socio-economic index (SESI) and the ecological index (ECSI). The ecological index has not yet been implemented for the pilot area, but the risk modelling system is prepared to include it, once data are available.

The characterization of the coastal sensitivity and socioeconomic indices in the pilot area (Portuguese continental coast) was made in the scope of the EROCIPS project. Along with desk work, based on Aerial photos and on Google Earth, field surveys were conducted of the whole Portuguese continental shoreline. This information is available on the web through Google Earth (MARETEC, 2007), and this KML format is directly imported to the developed risk assessment tool.

The vulnerability indices obtained for the pilot area were defined with a very high spatial discretization, dividing the shoreline into multiple segments or stretches in extensions that can be as small as $200 \mathrm{~m}$, realistically representing the variability of the shoreline.

\subsubsection{Coastal Sensitivity Index}

The CSI index represents the quantification, on a logarithmic scale, of the valuation of the environmental sensitivity (ecological, landscape) of the areas of the maritime coast and/or the surrounding waters that can be reached by sea pollution from hydrocarbons and/or other dangerous substance spills.

For the general group of areas of the maritime coast, NOAA's ESI (Environmental Sensitivity Index) was adapted for the Portuguese Continental Coast (modifications were related to the specificities of the Portuguese shoreline). The ranking of this index, which varies in the range 1-10, coincides with the scale of the NOAA's ESI (NOAA, 2002), defined to characterize zones of the shoreline as a function of the following parameters:

- exposure to wave and tidal energy;

- slope of the coast (intertidal zone);

- type of substrate (size, permeability and mobility);

- biological productivity and sensitivity;

- ease of clean-up.
The colours used to visualize the CSI ranking are the same as used in NOAA's ESI (a list description of CSI is included in Appendix A, Table A1).

In regions like coastal shoreline (restricted) waters, commercial ports, all-purpose terminals, fishing ports, marinas or yacht harbours and unrestricted waters, CSI is invariable and considered to be 6 . However, as this tool is only estimating risks of shoreline contamination, coastal vulnerability indices of restricted or unrestricted waters/open sea are not considered by the risk model.

The CSI values obtained in the pilot area vary from a range of 1-10, with an average value of 4.1 and a median value of 3.

\subsubsection{Socio-economic Index}

This index (SESI) intends to reflect the social-economic importance to the populations of the exploitation of the coastal zone under analysis (e.g. a beach not often used, or used but without significant infrastructures, and/or a beach with important economic value - restaurants, etc.). While the CSI already considers the normal habitats for that shoreline, it does not consider other improvements that can exist in the zone and that are not specific to the characterization of the CSI index, such as fisheries or aquaculture, that therefore have to be considered through the social-economic index SESI. This index varies from 1 to 5 (a complete list description of SESI is included in Appendix A - Table A2).

The SESI values obtained in the pilot area vary in the range of $1-5$, with an average value of 1.8 and a median value of 1 .

\subsubsection{Ecological Index}

The ECSI is used to consider special protected areas that are not included in the CSI. This index varies from 1 to 5 . Although the risk model has been prepared to include this ecological index, this has not been set up yet for the area of study - therefore, a constant value of 3 is now temporarily used as ECSI in all shoreline stretches. Currently a methodological definition of this index is being pursued in the scope of the ARCOPOL platform project.

\subsection{Metocean data}

Wind, current, waves and visibility are taken into account for the probability of an accident, which is modified with correction factors adjusted by those meteo-oceanic conditions. These parameters can be imported to the system's database in real time from online internal or third-party forecasting systems (as long as model output files are provided in native MOHID format - HDF5 - or in standard CF-compliant netCDF formats, available online in web servers - preferably FTP or THREDDS catalogue). The system implemented in this work imports MARETEC-IST's forecast regional solutions available online in http://forecast.maretec.org and http: //meteo.ist.utl.pt. 
Current and water properties (temperature and salinity) are obtained from the PCOMS-MOHID model (Mateus et al., 2012; Pinto et al., 2012). PCOMS is a 3-D hydro-biogeochemical model of the Iberian Western Atlantic region. Ocean boundary conditions are provided by the Mercator-Ocean PSY2V4 North Atlantic and by tidal levels computed by a 2-D version of MOHID (Neves, 2013; Ascione Kenov et al., 2014), forced by FES2004, and running on a wider region. PCOMS has a horizontal resolution of $6.6 \mathrm{~km}$ and a vertical discretization of 50 layers with increasing resolution from the sea bottom upward, reaching $1 \mathrm{~m}$ at the surface (Ascione Kenov et al., 2014).

Atmospheric conditions (wind velocity, surface air temperature, atmospheric pressure and visibility) are obtained from the meteorological forecasting system IST-MM5, using MM5 model (Grell et al., 1994) with a $9 \mathrm{~km}$ spatial resolution. This operational model was initially implemented by Sousa (2002), and updated in 2005 (Trancoso, 2012). This model is also used as atmospheric forcing of PCOMSMOHID.

The wave parameters (wave period, wave height, wave direction and wave length) are obtained from the Portuguese wave forecasting system implemented at MARETEC-IST, using the WaveWatchIII model (version 3.14 - Tolman, 2009 ) with a $5 \mathrm{~km}$ spatial resolution, and wind forcing provided by Global Forecasting System (GFS) from the National Oceanic and Atmospheric Administration (NOAA), with a spatial resolution of $0.5^{\circ}$ (Franz et al., 2014).

These meteo-oceanic properties are also used to feed the oil spill fate and behaviour model integrated in the system, which is used to estimate the hypothetical vessel-based spilled oil amount reaching shoreline.

\subsection{Oil spill model}

The integrated oil spill model used in this work is the MOHID oil spill fate and behaviour component, integrated in MOHID Lagrangian transport module, where simulated pollutants are represented by a cloud of discrete particles (or super-particles) advected by wind, current and waves, and spread due to random turbulent diffusion or mechanical spreading. The MOHID oil spill modelling component was initially developed in MOHID in 2001 (Fernandes, 2001), and over the years the model has been operationally applied in different incidents (Carracedo et al., 2006; Janeiro et al., 2014), field exercises and studies worldwide, allowing the simulation of all major oil transport and weathering processes at sea. The source code of the oil spill modelling system was recently updated to include full 3-D movement of oil particles, wave-induced currents and oil-shoreline interaction (Fernandes et al., 2013), as well as blowout emissions (Leitão, 2013).

This oil spill model has the ability to run integrated with the hydrodynamic solution, or independently (coupled offline to metocean models), this latter being the option adopted for integration in the developed dynamic risk tool, taking advantage of metocean models previously run, and thus optimizing the computational efficiency.

The oil spill model is freely available for public access, since it is integrated in the MOHID numerical modelling system which follows a FOSS (free/open source software) strategy.

The dynamic risk tool continuously runs the MOHID oil spill model to simulate hypothetical spills from multiple vessels across the coast, then taking into account the fraction of oil that would approach the coastline.

\subsection{Risk model}

Two different integrated risk types (they are integrated because they take into consideration different types of incidents) are computed: (a) risk of oil spill incident; (b) risk of shoreline contamination.

Both integrated risk types are variable in space and time due to variable vessel information and metocean conditions (that influence probability of an accident, as well as fate and behaviour of oil spills simulated). The simultaneous calculation of the risk posed by each vessel crossing a pilot area is integrated, allowing the generation of a dynamic shoreline risk map for that zone.

\subsubsection{Risk of oil spill incident}

The risk of oil spill incident quantifies the severity based on vessel dead weight tonnage and vessel position, with higher or lower risk if the vessel is navigating in restricted or unrestricted waters, respectively. This risk type does not take into consideration the effects on shoreline, and is represented in each vessel.

Different types of incidents are considered in the risk model: grounding, foundering and structural failures, collision (with a ship or with port facilities), fire and explosion, illegal and operational discharges. In order to obtain the integrated ship risk of spill incident, the partial probability and severity indices are integrated. Probability indices from the different types of incidents are summed, and a weighted average severity index from the different types of incidents is determined. The sum of the probability indices $\left(I_{\sum \text { PSI }}\right)$ with the weighted average severity index $\left(\overline{I_{\mathrm{SSI}}}\right)$ provides the integrated risk of spill incidents ( $\left.I_{\text {IRSI }}\right)$ :

$I_{\mathrm{IRSI}}=I_{\sum \mathrm{PSI}}+\overline{I_{\mathrm{SSI}}}$.

The detailed determination of $\overline{I_{\text {SSI }}}$ and $I_{\sum \text { PSI }}$ is explained in Appendix B.

\subsubsection{Risk of shoreline contamination}

The risk of shoreline contamination takes into account the interaction with the coastline, therefore the severity/shoreline consequences additionally include the virtual spilled oil frac- 
tion reaching shoreline and its environmental and socioeconomic vulnerabilities, instead of simply considering the vessel deadweight tonnage and position. The oil reaching shoreline is quantified with an oil spill fate and behaviour model that continuously simulates virtual oil spills from the vessels included in the domain. Alternatively, a "nonmodelled" shoreline contamination risk rating is computed, without using the oil spill model for the determination of shoreline impact - in this case, a vessel shoreline proximity correction factor is used and subtracted to the risk value (with this correction factor decreasing as the vessel approaches the coastline). This risk type is represented in shoreline stretches, taking into consideration the effects from multiple vessels affecting that zone. The division of shoreline stretches for characterization of shoreline contamination risk is based on the same division used in the coastal vulnerability characterization.

The shoreline contamination risks provided are in fact a percentile (by default, percentile 98, but can be customized) of the shoreline contamination risks determined from the different vessels. Shoreline contamination risks below a userdefined value are not considered.

\subsubsection{Probability}

The probability/frequency of occurrence of a specific type of incident in a ship leading to an oil spill is obtained from statistical constants (frequency of incidents per distance navigated, or annual incident frequency) corrected with a combination of a different factors identified as relevant in the generation of those incidents (e.g. visibility, currents, proximity to coast, etc.).

The choice of using the probability of incidents for each vessel per distance unit navigated was made because otherwise the annual frequency of accidents is too static, i.e. if hypothetically there is a ship anchored for an entire year, it will still provide a risk similar to a ship in circulation, which is not entirely true. A dynamic probability will be inevitably achieved using frequency of accidents per km navigated + distance navigated in a given period of time.

Generically, the probability of incident in a specific time period is computed as

$P=C \times \Delta S \times I$,

where $C$ is the frequency constant (accidents $\mathrm{km}^{-1}$ ), $\Delta S$ is the distance navigated by the ship (in $\mathrm{km}$ ), and $I$ are the multiplying correction factors.

The distance navigated by the ship is obtained directly by ship velocity (from AIS data) and time step for risk analysis (defined by the end-user).

Since illegal/operational discharges occur based on human decisions, their probability is not influenced by environmental conditions. Thus, no correction factors are applied to the calculation of this probability. Also in this type of incident, the probability is not based on incident frequency per dis- tance navigated, but on annual frequency - it is assumed that deliberate discharges occur independently of vessel speed. The probability of operational discharges $\left(P_{\mathrm{OD}}\right)$, is determined as follows:

$P_{\mathrm{OD}}=\frac{C_{\text {annual }}}{365} \times \Delta t$,

where $C_{\text {annual }}$ is the frequency constant (incidents per year) and $\Delta t$ is the time step used in the risk tool (in days).

A logarithmic scale from 1 to 8 was adopted for the index of probability. The correspondence between annual probability and index of probability can be represented by the following equation (derived from the Table $\mathrm{C} 1$ in Appendix $\mathrm{C}$ ), based on Filipe and Pratas (2007), and inspired by IMO recommendation (IMO, 2002):

$I_{\mathrm{PSI}}=\log \left(P_{\text {annual }}\right)+6\left(I_{\mathrm{P} \min }=0 ; I_{\mathrm{Pmax}}=8\right)$.

The annual probability $\left(P_{\text {annual }}\right)$ is based on

$P_{\text {annual }}=365 \times \frac{P}{\Delta t}$,

where $P$ is the probability obtained by the previously explained method, for a specific time step $\Delta t$ (in days).

\section{Frequency constants}

Different frequency/probability constants of incidents are included in the risk model as a way to include some differentiation based on type of incidents and some probabilistic data obtained from statistical information on past incidents. These values can be changed by the end-user at any time.

In this study, frequency constants of incidents per distance unit navigated are obtained from IAEA (2001), and missing constants are obtained from the combination of previous reports of the Lloyd's Register accidents database (the relationship between annual frequency constants was used to extrapolate frequency constants per distance navigated). The numerical values of the frequency constants used can be found in Appendix C (Table C2).

According to IAEA (2001), the frequency of incidents due to fire and explosion does not vary significantly with the region. Therefore, the frequency for this type of accident per distance navigated is kept constant.

Also in the same report, there is no reference to illegal/operational discharges. For this kind of incident, annual incident frequency is assumed, since these discharges are independent of vessel speed. It is also assumed that such discharges do not occur in restricted waters.

\section{Multiplying correction factors}

Multiplying correction factors are used to modify the probabilities of spill incidents based on metocean conditions (wind velocity, current velocity, wave height and visibility), proximity to coast and ship type. The correction factors are not 
Table 1. Summary of multiple correction factors used by each type of accident ( $I_{\text {curr }}$ : correction factor due to currents; $I_{\text {wind }}$ : correction factor due to wind; $I_{\text {prox }}$ : correction factor due to proximity to coast; $I_{\text {ship }}$ : correction factor due to ship type; $I_{\text {visib }}$ : correction factor due to visibility; $I_{\text {wave }}$ : correction factor due to waves).

\begin{tabular}{ll|ll}
\hline & Restricted waters & \multicolumn{1}{c}{ Unrestricted waters } \\
\hline $\begin{array}{l}\text { Type of } \\
\text { accident }\end{array}$ & Correction factors $(I)$ & Type of accident & Correction factors $(I)$ \\
\hline $\begin{array}{llll}\text { Ship-to-ship } \\
\text { collision }\end{array}$ & $I_{\text {curr }} \times I_{\text {wind }} \times I_{\text {prox }} \times I_{\text {ship }}$ & $\begin{array}{l}\text { Ship-to-ship } \\
\text { collision }\end{array}$ & $I_{\text {curr }} \times I_{\text {wind }} \times I_{\text {visib }} \times I_{\text {wave }}$ \\
\cline { 3 - 4 } $\begin{array}{lll}\text { Collision with } \\
\text { port facilities }\end{array}$ & & Foundering & $I_{\text {wave }} \times I_{\text {prox }}$ \\
\hline Grounding & $I_{\text {curr }} \times I_{\text {wind }} \times I_{\text {ship }}$ & $\begin{array}{l}\text { Grounding during } \\
\text { navigation }\end{array}$ & $I_{\text {curr }} \times I_{\text {wind }} \times I_{\text {visib }} \times I_{\text {wave }} \times I_{\text {prox }}$ \\
\cline { 3 - 4 } & & Drift grounding & $I_{\text {curr }} \times I_{\text {wind }} \times I_{\text {wave }} \times I_{\text {prox }}$ \\
\hline Fire/explosion & $I_{\text {curr }} \times I_{\text {wind }} \times I_{\text {prox }} \times I_{\text {ship }}$ & Fire/explosion & $I_{\text {curr }} \times I_{\text {wind }} \times I_{\text {prox }} \times I_{\text {ship }}$ \\
\hline
\end{tabular}

applied to the probability of having operational/illegal discharges because these incidents are considered deliberate or independent of (and not controlled by) external effects. The values used can also be changed or calibrated by the end-user.

The correction factors included by default in this study were obtained from the Risk Assessment Report for the Portuguese and Galician Coast - EROCIPS (Filipe and Pratas, 2007), and the values used are listed in Appendix C (Tables $\mathrm{C} 3$ and $\mathrm{C} 4)$. Table 1 summarizes the multiple correction factors used by each type of accident.

\section{Minimum risk/minimum probability}

A minimum or residual probability of an accident per unit time must be assumed, to avoid the determination of null or (nearly null) probabilities when vessels are anchored or moving very slowly (because the risk model computes the incident probability based on ship velocity). Even at slow motion or stopped, a ship has always a risk of a spill accident. For instance, there is still a chance of collision with another ship, or to anchor in a danger zone and eventually generate a grounding accident (depending on the weather and oceanographic conditions).

This probability is obtained as a function of a minimum velocity. Below this velocity value, the vessel is assumed to have a constant accident probability. The minimum velocity is user defined, and by default the value of $0.36 \mathrm{~m} \mathrm{~s}^{-1}$ was adopted (selection based on the minimum value corresponding to the lower correction factor for current velocity).

\subsubsection{Severity}

The severity index list of hydrocarbon and other hazardous substances spills, whether in open sea or in restricted waters due to the various types of accidents, follows IMO recommendations (IMO, 2002) and is described in Filipe and
Pratas (2007). A logarithmic scale from 1 to 8 was adopted, following the same scale as the probability index (Table D1 in Appendix D gives details of the severity index).

\section{Severity of risk of spill incident}

The severity in the risk of spill incident varies with the ship position (restricted/unrestricted waters), and with the hypothetical amount of spilt product. Typical values of amount of oil spilt are estimated based on the ship type, weight and the type of incident, in order to estimate the severity index of spill incident $\left(I_{\mathrm{SSI}}\right)$ according to the values in Filipe and Pratas (2007). Further detailed information on the formulations used are listed in Appendix D, Tables D2 and D3.

\section{Severity of risk of shoreline contamination}

As mentioned before, the risk of shoreline contamination from each vessel considers the risk of spill incidents plus the interaction with the coast, taking into consideration the coastal vulnerability, and the potential contamination of the near-shore. This potential contamination is computed by two different approaches: by estimating the oil fraction reaching the coastline - a method herein called the "modelled" risk of shoreline contamination; or alternatively by a correction factor based on ship distance to coastline - a method herein called the "non-modelled" risk of shoreline contamination.

In both approaches (modelled and non-modelled), the computed severity index of shoreline contamination $\left(I_{\mathrm{SSC}}\right)$ includes the severity index of risk of spill incident $\left(I_{\mathrm{SSI}}\right)$ mentioned in the previous section, with a weight of $50 \%$. The remaining $50 \%$ of severity is obtained from the coastal vulnerability index $\left(I_{\mathrm{V}}\right)$, as expressed by

$I_{\mathrm{SSC}}=0.5 \cdot I_{\mathrm{SSI}}+0.5 \cdot I_{\mathrm{V}}$ 
where the coastal vulnerability index can be represented as an arithmetic mean of the different coastal vulnerability indices:

$I_{\mathrm{V}}=\frac{8}{5} \cdot\left(\frac{0.5 \mathrm{CSI}+\mathrm{SESI}+\mathrm{ECSI}}{3}\right)$.

The fraction $8 / 5$ is used to convert the vulnerability index scale (from $1-5$ to $1-8$ ), to the same scale adopted in the severity of spill incident, as well as in the probability index. CSI is multiplied by 0.5 to convert the scale from 1-10 to 1-5 (as adopted in SESI and ECSI).

For non-modelled risk, a vessel shoreline proximity correction factor is subtracted to the severity of spill incident index (with this correction factor decreasing as the vessel approaches the coastline)

$I_{\mathrm{SSC}(\text { non-modelled) }}=I_{\mathrm{SSC}}-F_{\mathrm{SS}}$ with $F_{\mathrm{SS}} \leq I_{\mathrm{SSC}}$.

The determination of this factor depends on distance between spill site and shoreline, and on type of oil product/ship type (further details are in Table D4 in Appendix D).

For modelled risk, a modified severity of spill incident is adopted, in a more complex and realistic approach to determine the impact risk of oil spills on the shoreline, since fate and behaviour of oil spilled is taken into account, using the MOHID oil spill model, as described in Sect. 2.6. The modified severity of spill incident is obtained by using the regular equation for severity of spill incident in restricted waters (Appendix D, Table D3), but with a modified amount of oil spill $\left(Q^{*}\right)$ used instead of $Q$, computed as follows:

$Q^{*}=\frac{Q \times M}{L_{\text {stretch }}} \times L_{\text {unit }}$,

where $M$ is the modelled ratio of oil reaching near the shoreline stretch in a user-specified time period, $L_{\text {stretch }}$ is the shoreline stretch extension (m), and $L_{\text {unit }}$ is the shoreline stretch extension unit used (by default $1000 \mathrm{~m}$, but the enduser can change this value). $Q$ is the amount of oil, based on ship type, weight and the type of incident. Thus, $Q^{*}$ is the maximum amount of oil spilled reaching near the shoreline stretch per shoreline extension unit, in a certain time period. An increase in $L_{\text {unit }}$ will generate higher severity indices, so this value needs to be properly calibrated.

The quantification of modelled maximum oil contaminating a specific shoreline stretch is based on the maximum amount of oil present inside an area near the shoreline stretch. The definition of this "near-shore" area for each shoreline stretch is based on the distance to the shoreline stretch; thus, if the modelled oil reaches this near-shore area, it is assumed as relevant to the quantification of shoreline contamination risk. The near-shore distance is user defined, and by default it has a value of $2000 \mathrm{~m}$ from the coast. The time period used in the quantification of maximum oil spilled reaching near the shoreline stretch has a default value of $24 \mathrm{~h}$ (configurable). Updates and new oil spill simulations from
Table 2. Risk matrix based on probability and severity indices, with corresponding representation with colour. $2<I_{\mathrm{RSI}} \leq 5$ (normal text): dark green - very low or insignificant risk; $6 \leq I_{\mathrm{RSI}} \leq 7$ (italic text): light green - low or minor risk; $8 \leq I_{\mathrm{RSI}} \leq 9$ (bold text): yellow - medium or moderate; $10 \leq I_{\mathrm{RSI}} \leq 11$ (underline text): orange - high level or serious; $12 \leq I_{\mathrm{RSI}}<16$ (underline + bold text): red - very high or critical).

\begin{tabular}{|c|c|c|c|c|c|c|c|c|c|}
\hline \multicolumn{2}{|c|}{ Risk index $\left(I_{\mathrm{RSI}}\right)$} & \multicolumn{8}{|c|}{ Severity index $\left(I_{\mathrm{SSI}}\right)$} \\
\hline \multirow{9}{*}{ 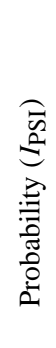 } & & 1 & 2 & 3 & 4 & 5 & 6 & 7 & 8 \\
\hline & 1 & 2 & 3 & 4 & 5 & 6 & 7 & 8 & 9 \\
\hline & 2 & 3 & 4 & 5 & 6 & 7 & 8 & 9 & $\underline{10}$ \\
\hline & 3 & 4 & 5 & 6 & 7 & 8 & 9 & $\underline{10}$ & $\underline{11}$ \\
\hline & 4 & 5 & 6 & 7 & 8 & 9 & $\underline{10}$ & $\underline{11}$ & $\underline{12}$ \\
\hline & 5 & 6 & 7 & 8 & 9 & $\underline{10}$ & $\underline{11}$ & $\underline{12}$ & $\underline{\mathbf{1 3}}$ \\
\hline & 6 & $\gamma$ & 8 & 9 & $\underline{10}$ & $\underline{11}$ & $\underline{12}$ & $\underline{13}$ & $\overline{14}$ \\
\hline & 7 & 8 & 9 & $\underline{10}$ & $\underline{11}$ & $\underline{12}$ & $\underline{\overline{13}}$ & $\underline{14}$ & $\underline{\overline{15}}$ \\
\hline & 8 & 9 & $\underline{10}$ & $\underline{\overline{11}}$ & $\underline{\overline{12}}$ & $\underline{13}$ & $\overline{14}$ & $\underline{\mathbf{1 5}}$ & $\underline{\overline{16}}$ \\
\hline
\end{tabular}

updated vessel positions are made every hour (this value is also configurable). The oil spill model simulations are made assuming always the same oil product released. The oil product included in the risk model (Carpinteria, medium oil from Group III) was chosen based on the profile of being a "worstcase scenario" for shoreline contamination, being a crude product from oil group III with low weathering effects along time.

\subsubsection{Risk matrix}

The risk matrix is the result of crossing both probability and severity indices, in order to obtain a risk rating - see Table 2 . The sum of both indices generates a risk index classification scale between 2 and 16 . These values are categorized with different risk levels and corresponding colours.

Independently of the integrated risk types applied (e.g. risk of spill incident; modelled risk of shoreline contamination; non-modelled risk of shoreline contamination), the same risk matrix should be applied.

In the case of shoreline contamination risk, at the present stage of the work, the visualization of risk values in the implemented software tool follows a continuous risk scale (bounded by the same limits as defined in the risk matrix categorization scheme), instead of a categorized scale, and using a different colour pattern from that proposed in Table 2. This option facilitates the visualization of variability in shoreline risk levels during the development period. In the future, the visualization of this risk level will be updated to the categorized view and using the same colour pattern as defined and presented in Table 2.

No risk acceptance/tolerability criteria were defined in the present work. 


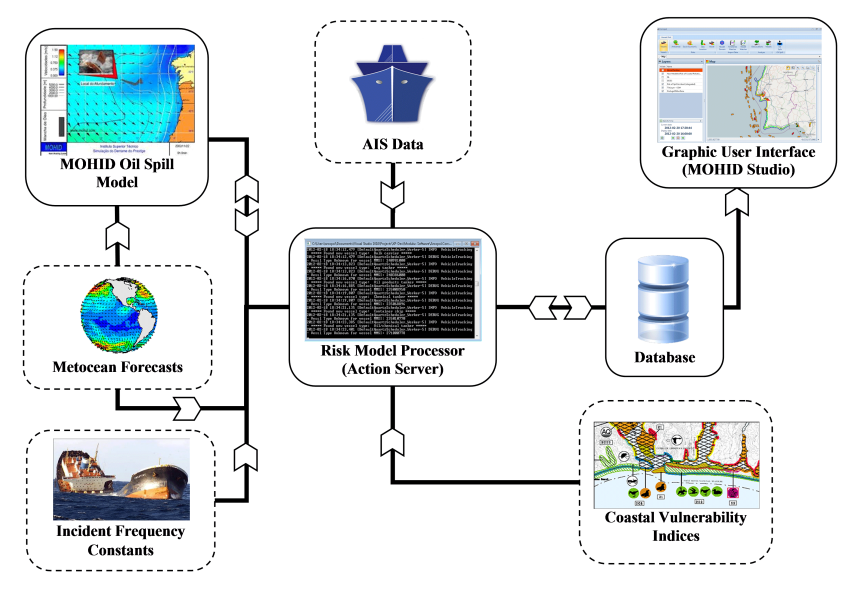

Figure 2. General information workflow in the risk modelling system.

\subsection{Development of software}

This risk assessment methodology has been implemented as a plugin from MOHID Studio, which is a GIS desktop interface that can also be used to run the MOHID water modelling system. MOHID Studio is a commercial platform property of Action Modulers, and has been entirely developed in c\#.NET language, using SQL Server components and the MOHID model.

The main philosophy of the software architecture was to create separate layers, allowing distributed tasks in different processes or computers, and a lighter graphic user interface (GUI). The general information workflow in the software framework is presented in Fig. 2. According to this, the main software framework is composed of four main components, exchanging information between them:

- an SQL Server or SQL Lite database, where all the data and meta-data are stored (metocean model outputs are not stored; only indexed);

- a desktop service (Action Server), which is continuously loading/ downloading updated data from different data sources (AIS data, metocean model outputs, etc.), managing the MOHID oil spill model, processing all information (and computing risk levels) and storing data on the database;

- MOHID oil spill project/executable file, which is continuously generating and running virtual oil spill simulations based on ship positions, and on instructions managed by the Action Server desktop service.

- A GUI (MOHID Studio), directly connected to the database, and showing requested data to the end-user. MOHID Studio can also be used to configure Action Server, and to run the on-demand risk assessment tool for specific periods.
MOHID Studio and Action Server do not need to be running on the same computer. The software architecture has also been developed to enable the publication of real-time risk mapping data in external platforms, including WMS layers, to facilitate the interoperability of the system.

\section{Results}

In this section, the response of the proposed risk model to different metocean conditions is evaluated in the pilot area, and the GUI developed in this work is also presented. Since the dynamic risk tool is capable of running in real time or on demand (for historical periods or virtual scenarios), it is assumed that a dynamic behaviour and proper response to the different variables means that the developed tool is also ready and able to provide results in an operational way.

\subsection{Graphic User Interface}

The risk modelling tool is able to run in continuous mode, allowing the user to follow in real time the ship traffic and specific vessel details, the evolution of risks crossed with background dynamic web maps (e.g. Google maps, Bing Maps, Open Street Maps) and many other geographic layers and features (Fig. 3) - e.g. visualizing metocean fields, topography, running oil spills on-demand, etc. When zooming the view, it is possible to check the very high level of resolution of the vulnerability indices and the associated risk levels being computed (Fig. 4).

\subsection{Ship incident risk}

Metocean conditions have a direct effect on risk of ship incident, because they can influence the probability of an accident occurring, according to the methodology proposed. These effects are included in the risk model through categorized correcting factors based on the range of metocean conditions.

One of the exercises performed in this study was to analyse the evolution of ship incident risks according to some of these metocean conditions used, organized in the same classes as the ones used in the correcting factors. In Fig. 5, ship incident risk levels are shown in different colour classes for different instants, together with wave model data (Fig. 5a and b) and wind speed (Fig. 5c and d) used in the risk model. Generally, the lower ship incident risk levels (in green) are present in ships crossing geographical areas where wind or wave conditions belong to lower classes. The same behaviour can be seen for vessels with higher incident risk levels - they tend to be determined in vessels crossing areas where wind speed or significant wave height are greater. It is also clear in Fig. 5 that the presence of a ship in different wave classes can contribute more significantly to different risk levels than wind speed - this is due to the fact that the wind multiplying correcting factor varies from 0.8 to 2 , while the wave 


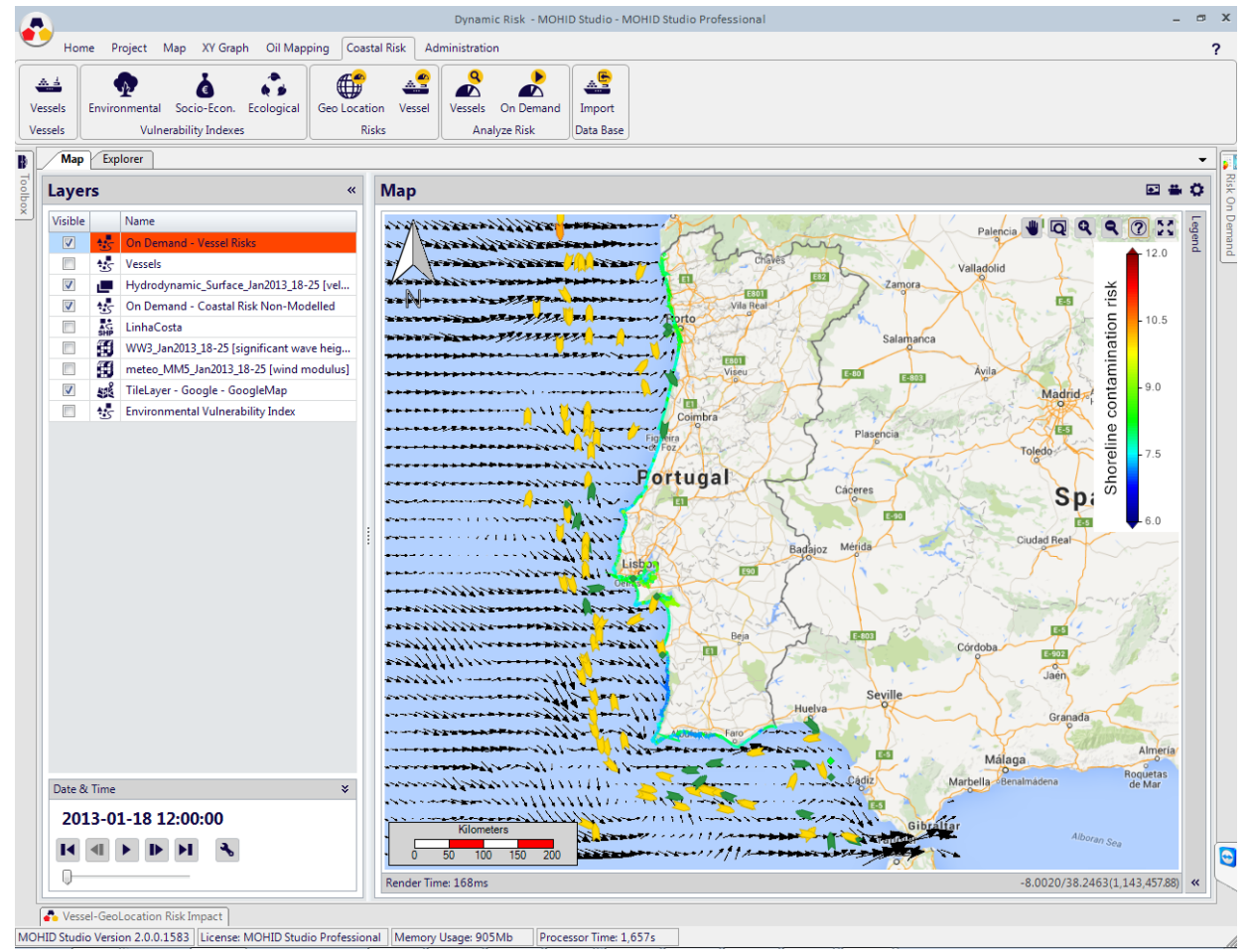

Figure 3. GUI layout, with simultaneous visualization of ship incident risks, shoreline contamination risks, surface water velocity and Google map layer. Ship incident risk colours are presented in categorized view (green, yellow, orange and red).

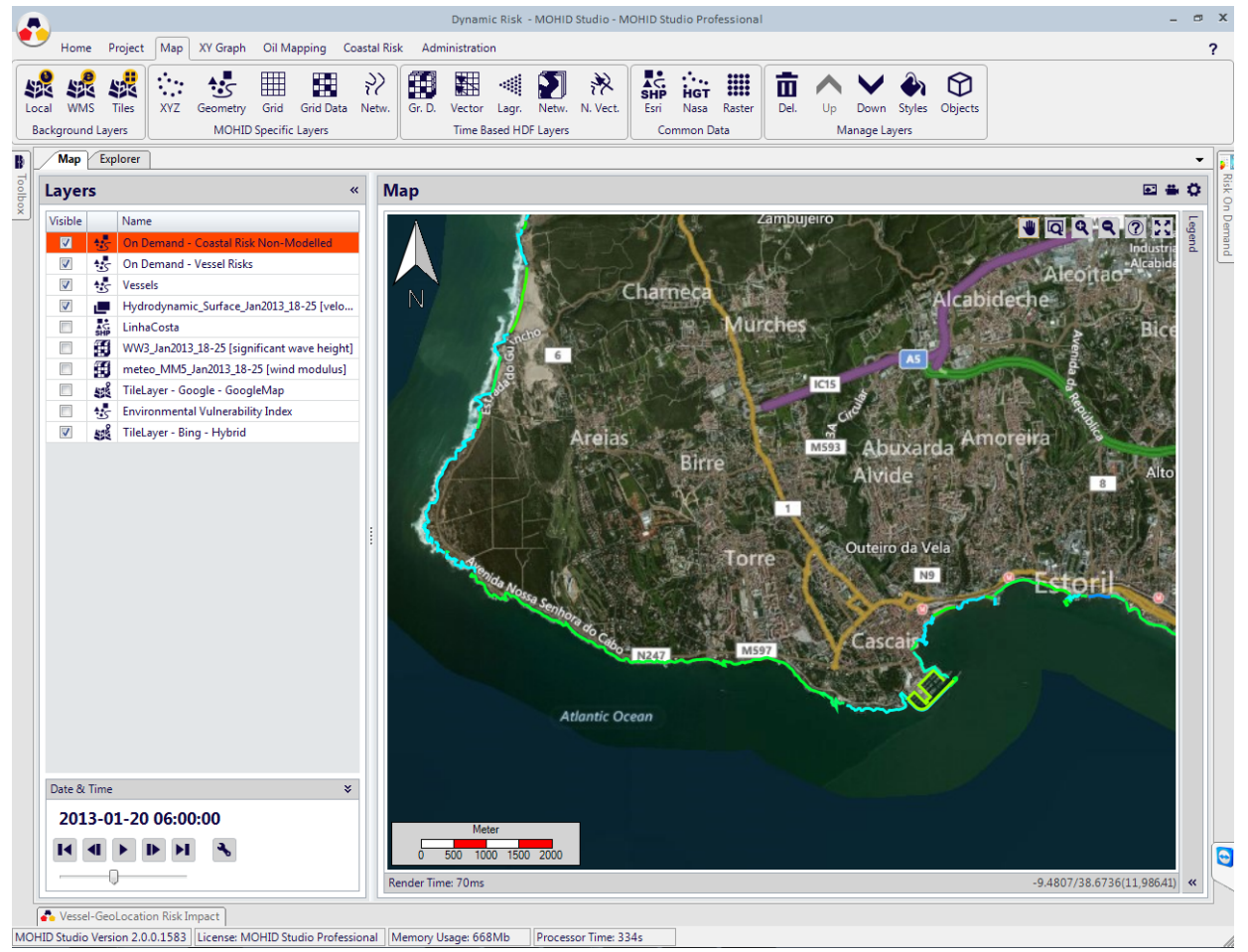

Figure 4. Close-up image of the GUI for the Lisbon area - simultaneous visualization of coastal sensitivity index and Bing Hybrid map layer. 


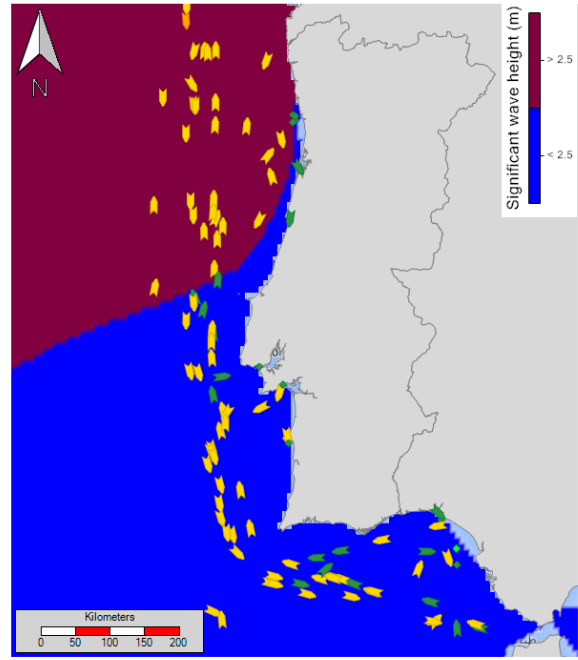

(a)

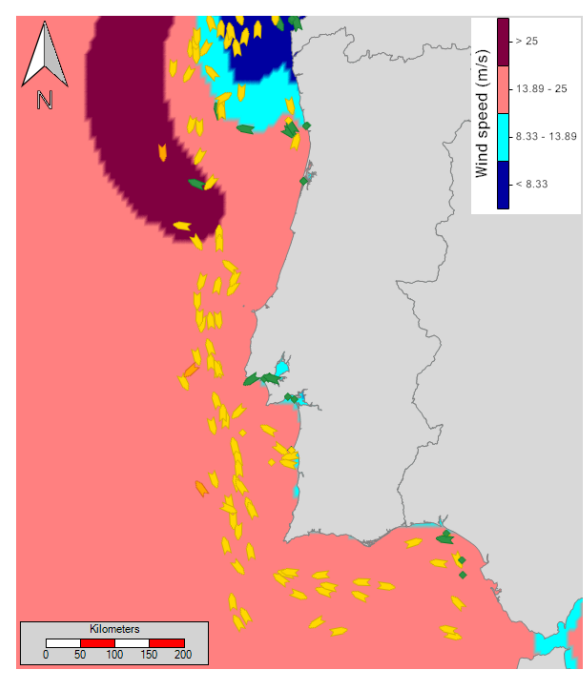

(c)

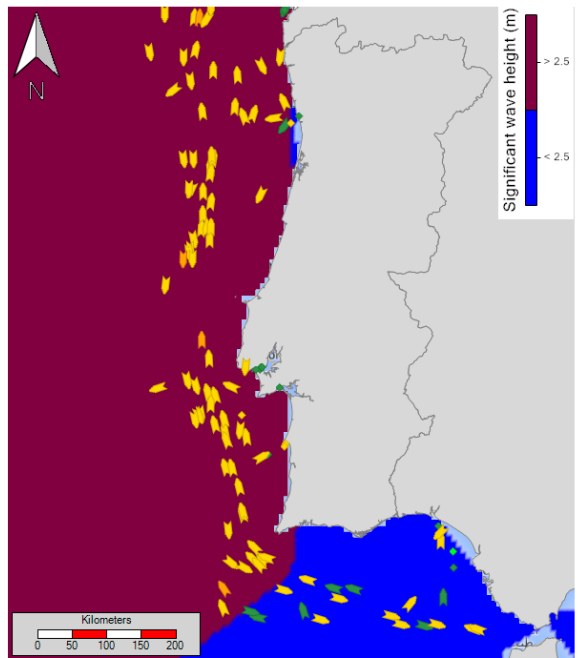

(b)

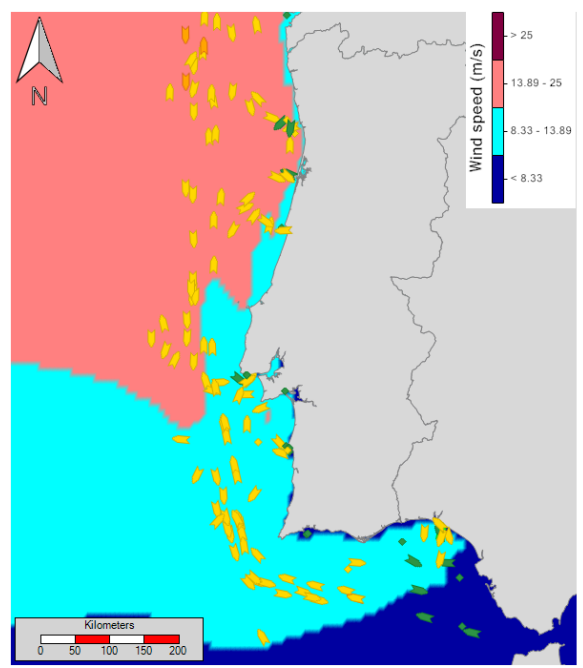

(d)

Figure 5. Ship incident risk levels (green ships mean lower risk, yellow means medium risk and orange ships mean higher risk) in the pilot area with background metocean conditions used. (a and b) Significant wave height on 18 January 12:00 h and 19 January 2013 00:00 h respectively; (c and d) wind speed on 19 January 06:00 h and 22 January 2013 06:00 h, respectively.

correcting factor used varies from 0.1 to 1 or 0.22 to 1.78 (detailed values of correcting factors used can be found in Appendix C).

A better evaluation of the importance of metocean conditions in the risk model can be tested using different metocean conditions for the same ship positions. Figure 6 illustrates the ship incident risk levels using different metocean conditions (6 months later), and exactly the same ship information as used in Fig. 5. Figure 6 clearly shows the dynamic change of risk levels directly affected by the wind and waves, for the same vessel traffic. Comparing Figs. 5 and 6, different ship risk levels can be observed. The effects of the other environ- mental conditions (visibility and surface water velocity) are similar to the properties illustrated here.

\subsection{Shoreline contamination risk}

When compared with ship incident spills, the evaluation of shoreline contamination risk from spills is more complex, as this parameter depends additionally on the coastal vulnerability indices, and is a result of an integration of risks from the different ships affecting each shoreline stretch. While it is easy to find different shoreline risk levels along the coast (e.g. Fig. 3), it can be difficult to evaluate, isolate and study the dependence of risk model on the multiple factors - for instance, metocean conditions, vessel traffic conditions, coastal 


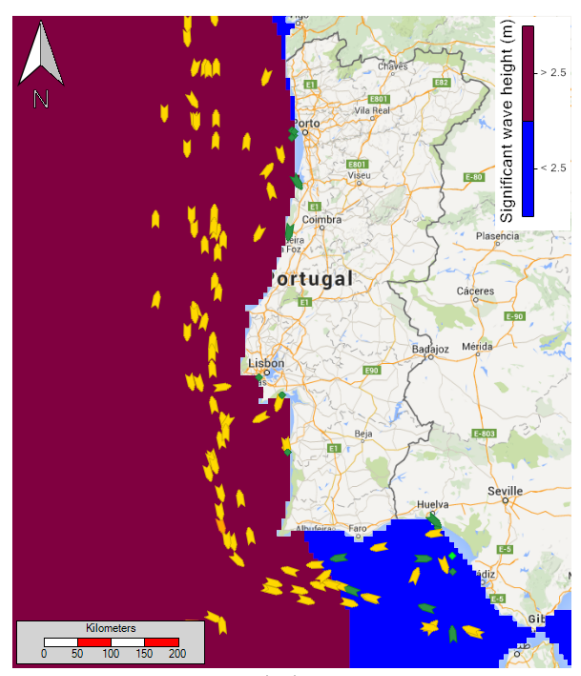

(a)

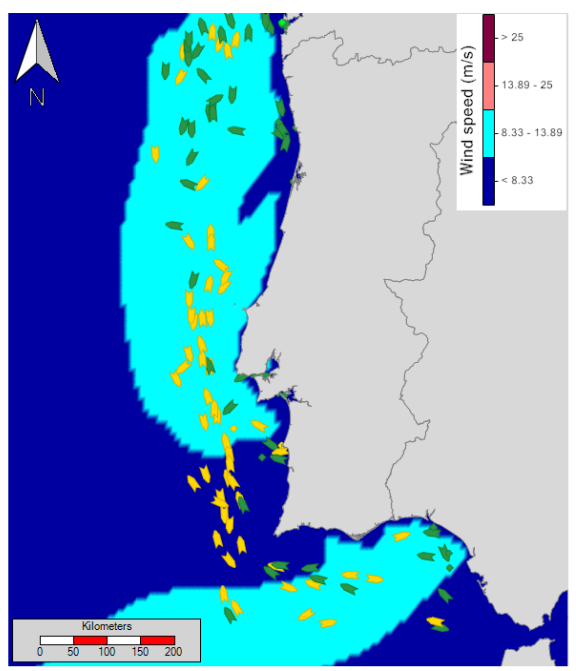

(c)

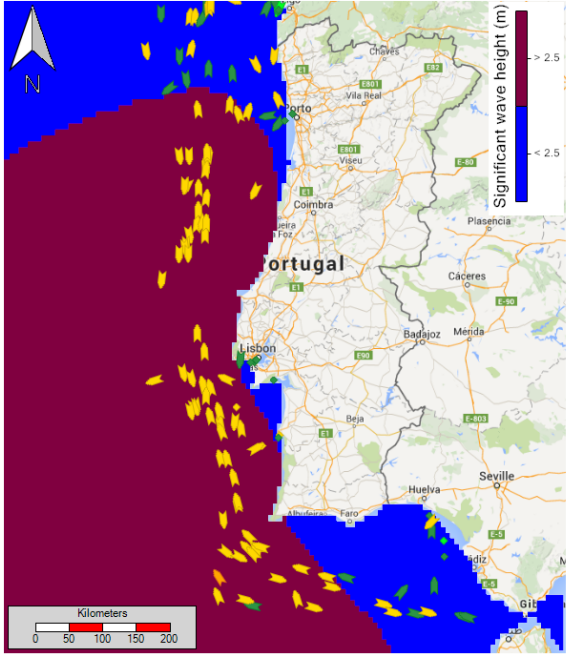

(b)

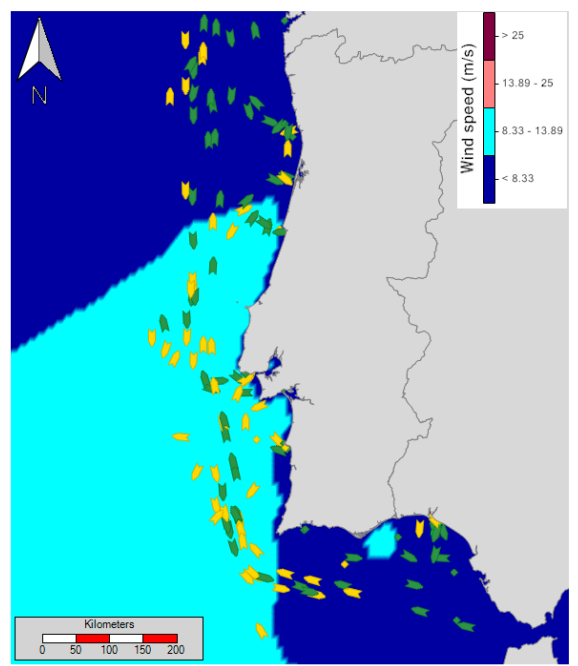

(d)

Figure 6. Ship incident risk levels (green ships mean lower risk, yellow means medium risk and orange ships mean higher risk) in the pilot area with background metocean conditions used. Computation is made with same vessel positions as used in Fig. 5. (a and b) Significant wave height on 18 June 12:00 h and 19 June 2013 00:00 h respectively; (c and d) wind speed on 19 June 06:00 h and 22 June 2013 06:00 h, respectively.

vulnerability, oil transport and weathering module. To facilitate the analysis, we start by evaluating the risk model behaviour without integrating the oil transport and weathering module (this matter is studied in Sect. 3.4).

\subsubsection{Evaluating dynamic response to vessels in the proximity}

In order to achieve this objective, an initial study was performed, with the selection of two different locations with exactly the same coastal vulnerability (Fig. 7), and subject to the same metocean conditions along the simulation. Therefore, the shoreline contamination risk levels are only influ- enced by the different vessels in the proximity. The results were generated, based on the registered vessel positions from two 1-week periods (between 18 and 25 January 2013 and between 18 and 25 June 2013), generating model risk outputs every $6 \mathrm{~h}$. The metocean conditions were defined as constant in the whole model domain along the two simulation periods. Typical winter (rough) and summer (calm) conditions were defined for January and June periods, respectively. Winter conditions: surface current velocity, $0.55 \mathrm{~m} \mathrm{~s}^{-1}$; wind velocity, $15 \mathrm{~m} \mathrm{~s}^{-1}$; significant wave height, $3 \mathrm{~m}$. Summer conditions: surface current velocity, $0.25 \mathrm{~m} \mathrm{~s}^{-1}$; wind velocity, $5 \mathrm{~m} \mathrm{~s}^{-1}$; significant wave height, $1.5 \mathrm{~m}$. 


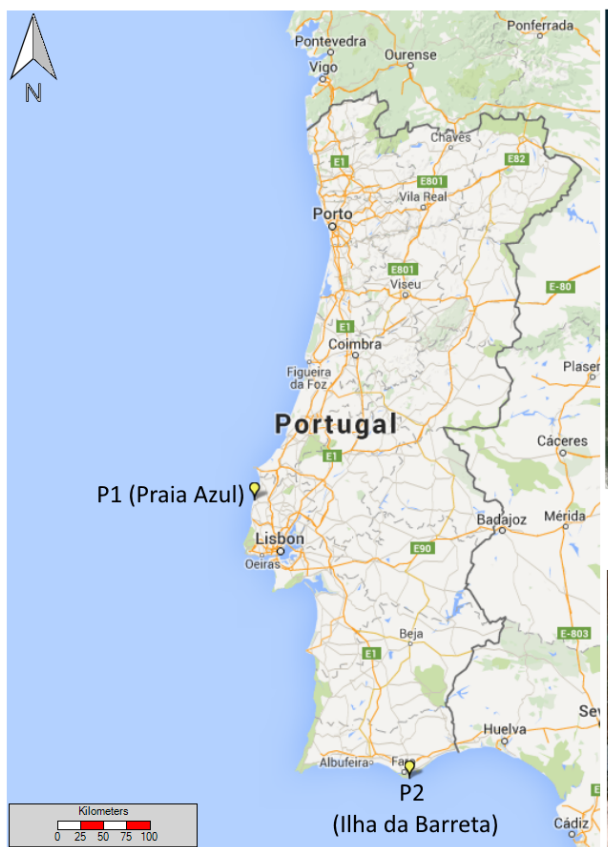

(a)

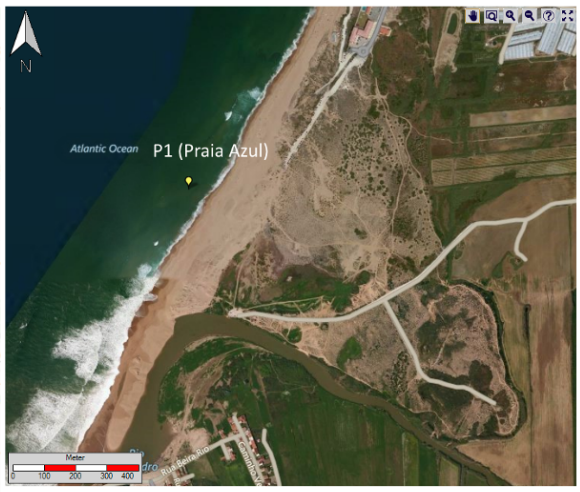

(b)

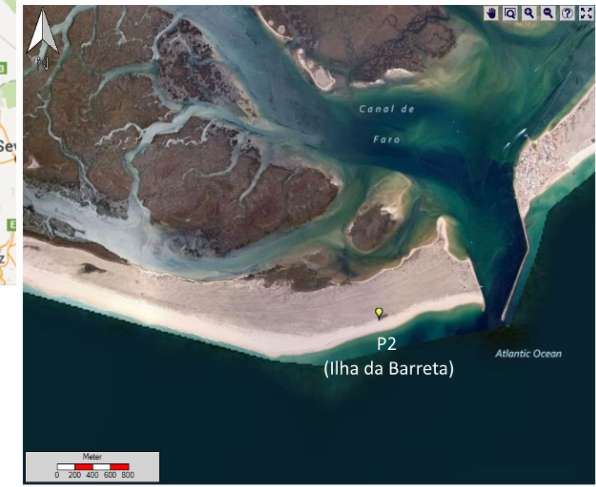

(c)

Figure 7. Location points for the shoreline contamination risk detailed study. (a) Location map; (b) aerial view from $\mathrm{P}_{1}$ (Praia Azul) and (c) aerial image from $\mathrm{P}_{2}$ (Ilha da Barreta).

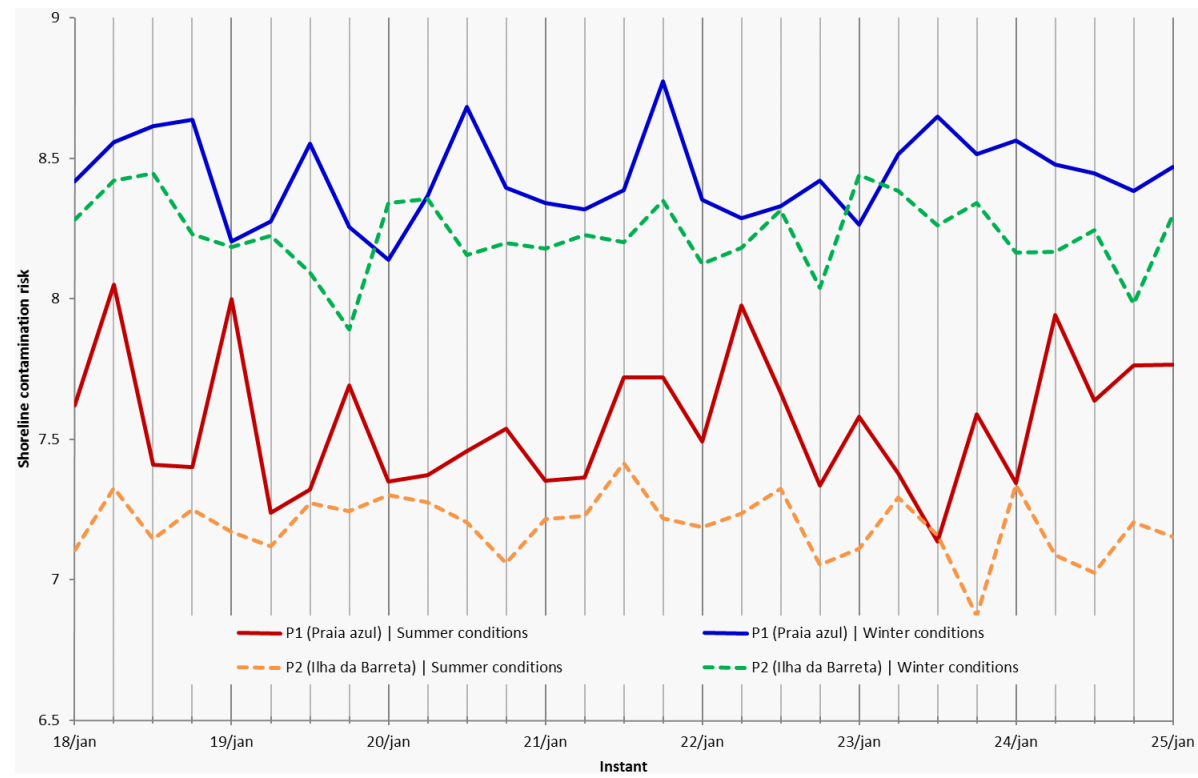

Figure 8. Evolution of shoreline contamination risk in $\mathrm{P}_{1}$ and $\mathrm{P}_{2}$ with vessel AIS information obtained between 18 and 25 January 2013 , using different space and time constant metocean conditions. Winter/rough conditions: surface current velocity, $0.55 \mathrm{~m} \mathrm{~s}{ }^{-1} ;$ wind velocity, $15 \mathrm{~m} \mathrm{~s}^{-1}$; significant wave height, $3 \mathrm{~m}$. Summer/calm conditions: surface current velocity, $0.25 \mathrm{~m} \mathrm{~s}^{-1}$; wind velocity, $5 \mathrm{~m} \mathrm{~s}{ }^{-1}$; significant wave height, $1.5 \mathrm{~m}$. 

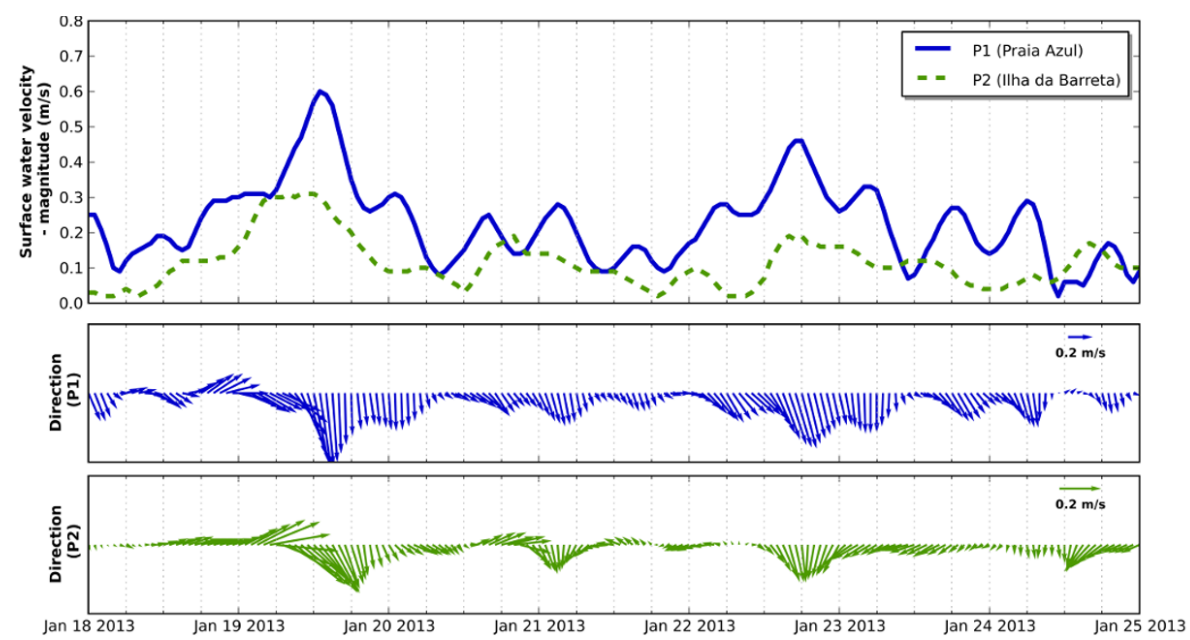

(a)
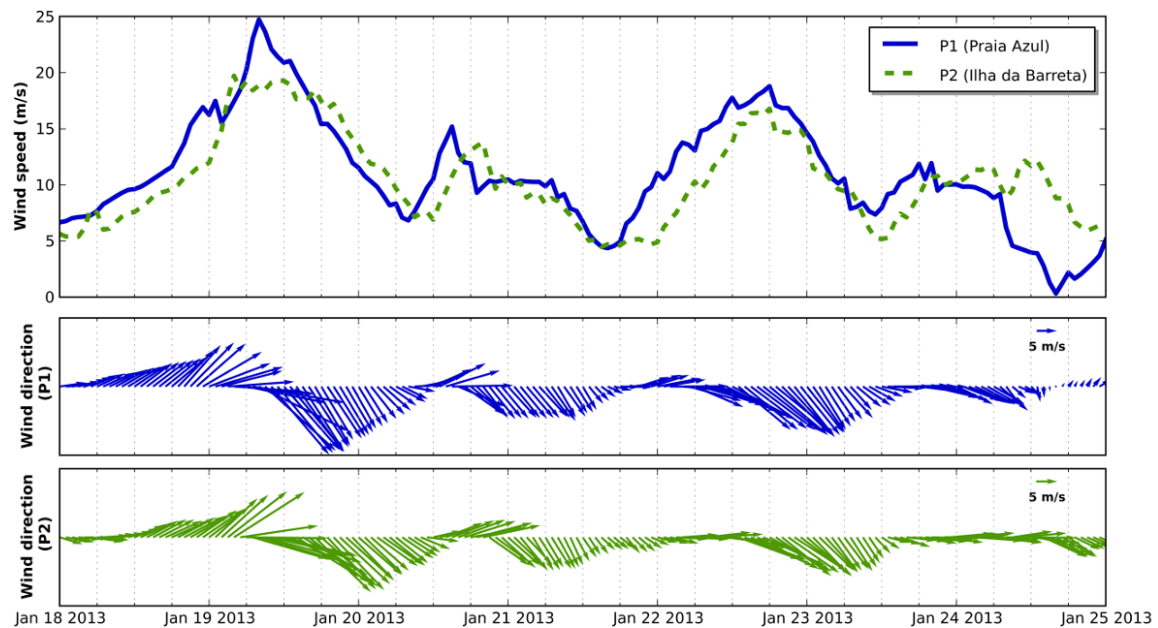

(b)

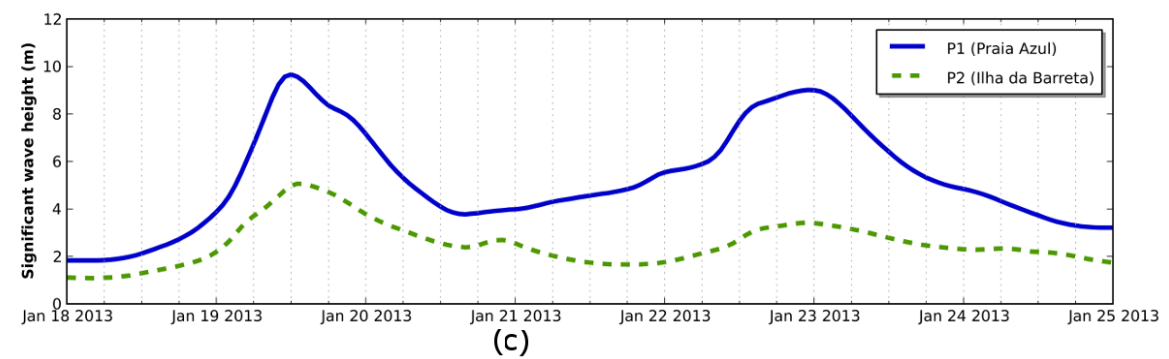

Figure 9. Metocean conditions used in the risk model, at points $\mathrm{P}_{1}$ and $\mathrm{P}_{2}$, during January. Surface water velocity, wind velocity and significant wave height.

As can be seen in Fig. 8, differences could be found when comparing both points for the same metocean conditions. The differences in risk values for both points subject to the same metocean conditions can only be explained by the different vessels in the proximity (with $\mathrm{P}_{1}$ having higher vessel traffic density in the neighbourhood), demonstrating the dynamic response of the risk model to vessel traffic, because this is the only factor changing along the time.
Additionally, Fig. 8 also illustrates the differences in risk values when comparing winter vs. summer conditions for the same point, justified by the response of the risk model to the metocean conditions. This aspect is further explained in what follows. 

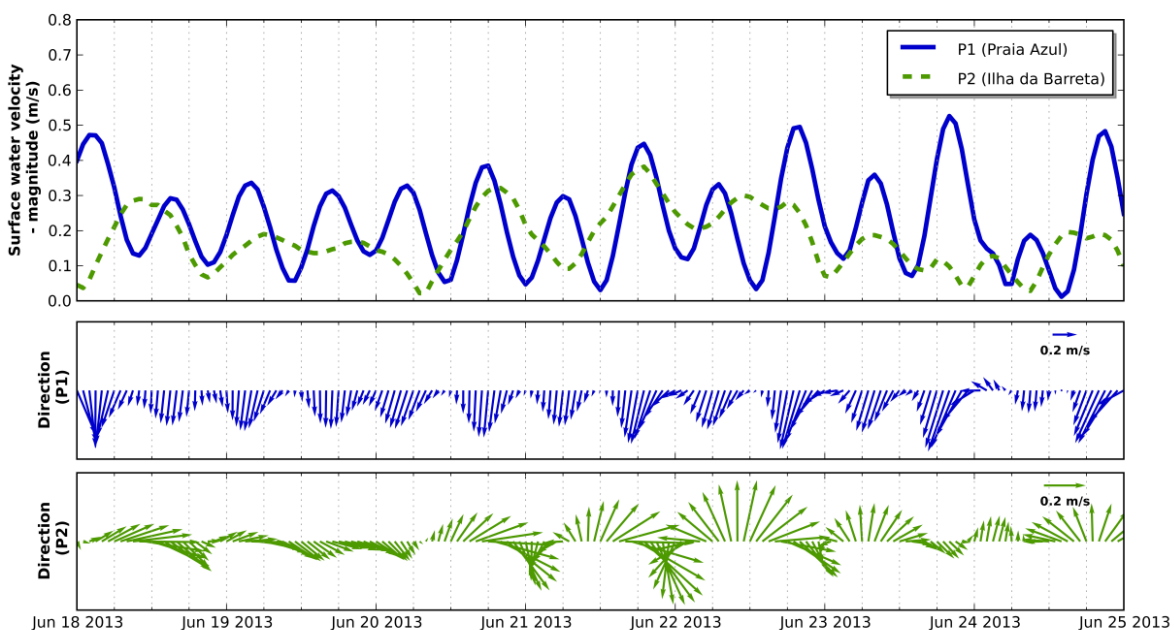

(a)
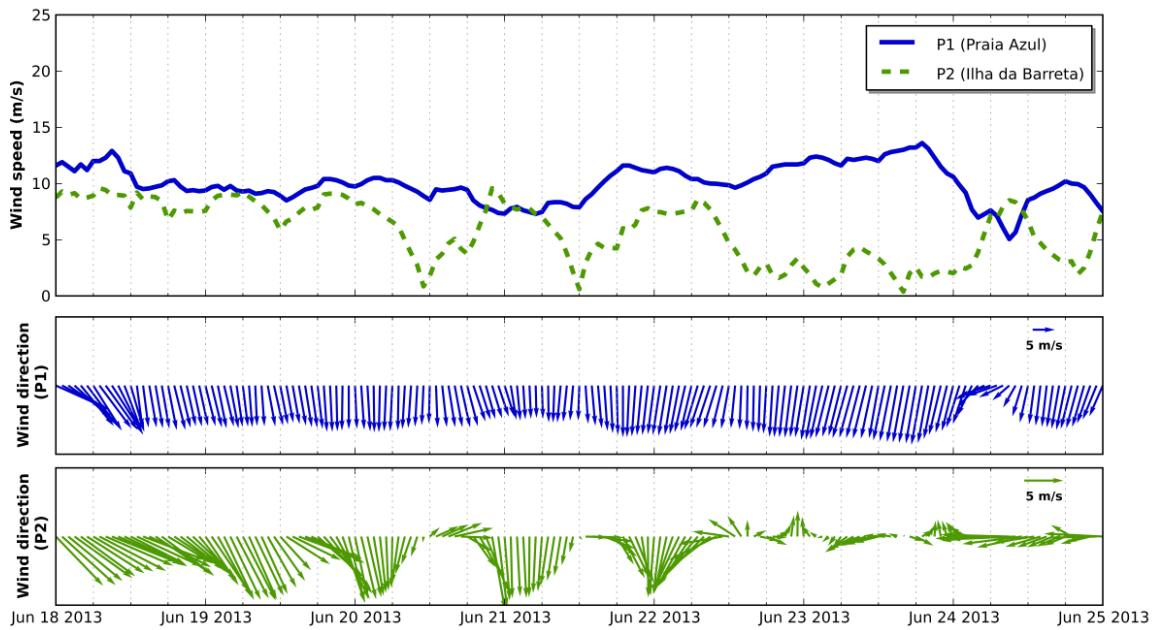

(b)

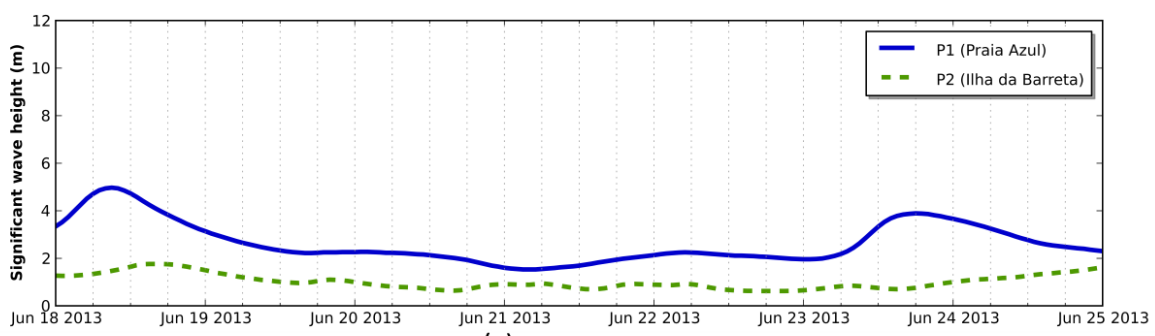

(c)

Figure 10. Metocean conditions used in the risk model, at points $\mathrm{P}_{1}$ and $\mathrm{P}_{2}$, during June. Surface water velocity, wind velocity and significant wave height.

\subsubsection{Evaluating dynamic response to metocean conditions}

Since the developed system is able to digest variable metocean conditions from forecasting systems, a second exercise with two simulations (winter: 18-25 January and summer: 18-25 June 2013) was performed for the same points as considered in the previous study $\left(\mathrm{P}_{1}\right.$ and $\left.\mathrm{P}_{2}\right)$, but here using metocean forecasts variable in time and space, and keeping the same variable vessel positions in both simulations (vessel's AIS positions obtained on 18-25 January 2013). Both shore locations are therefore subject to different metocean conditions and different vessels in the proximity, which can affect the evolution of shoreline contamination risk along time. The metocean conditions (from the models described in Sect. 2.5) used in both locations are illustrated in 


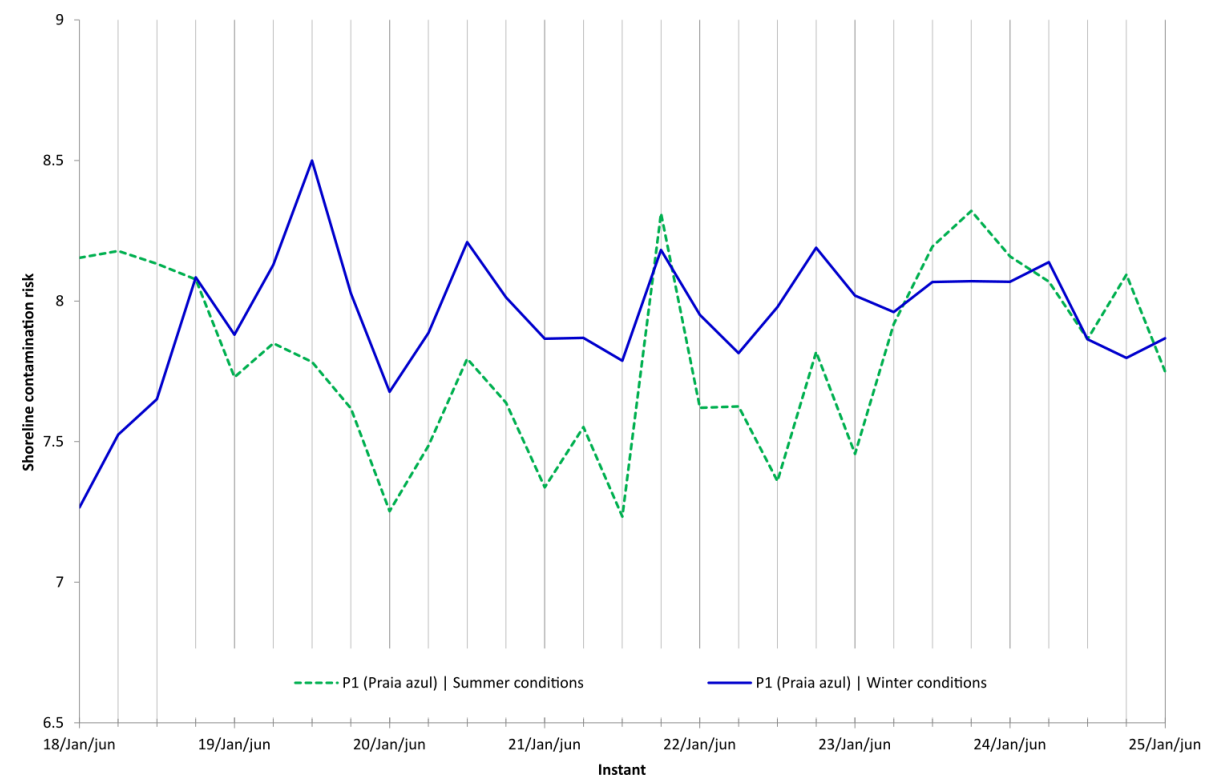

(a)

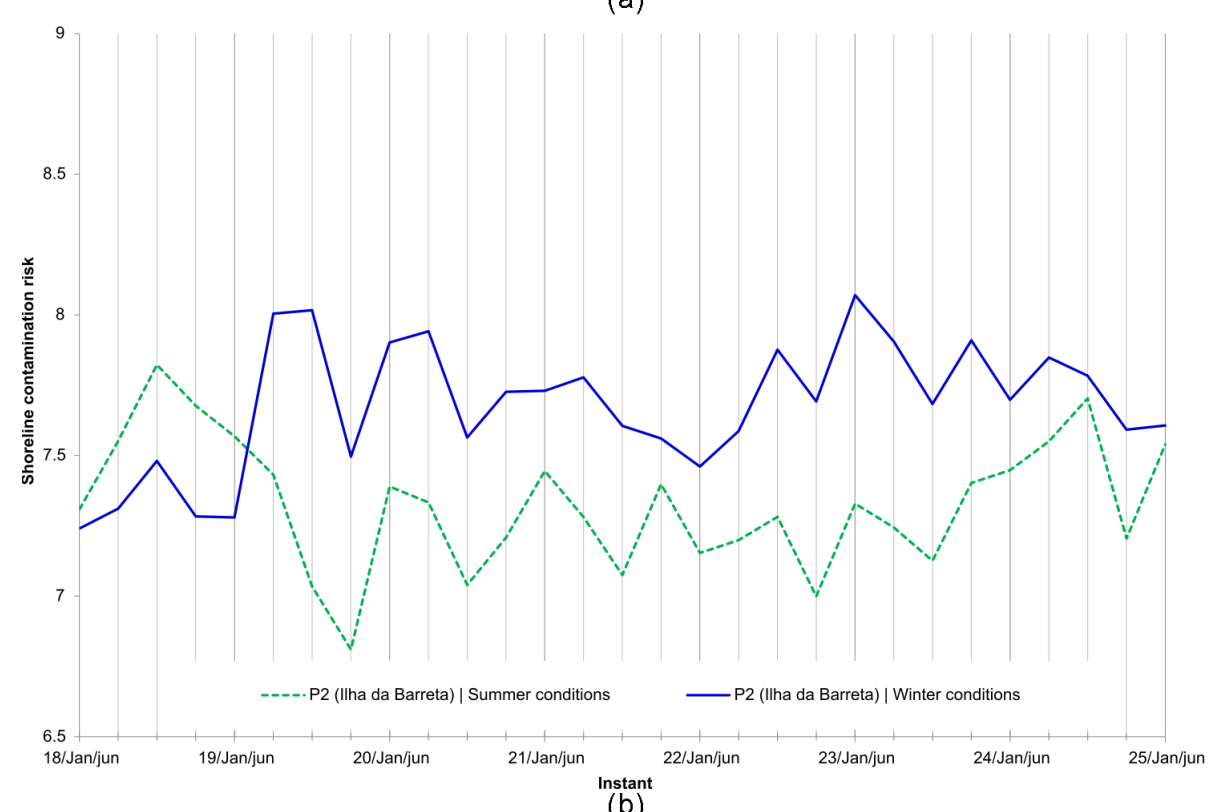

(b)

Figure 11. Evolution of shoreline contamination risk at $\mathrm{P}_{1}$ (a) and $\mathrm{P}_{2}$ (b) with vessel AIS information obtained between 18 and 25 January, 2013, and using different space and time variable metocean conditions, illustrated in Figs. 9 and 10.

Figs. 9 and 10. The results from this exercise are illustrated in Fig. 11.

The temporal variations and differences between shoreline contamination risk levels in winter and summer conditions identified can only be explained by the variation in metocean model conditions, since all the other conditions were kept constant. In general, risks are higher in January for both points, as expected (due to winter metocean conditions). The obtained results show risk variations of $0.5-1$ risk units in a $6 \mathrm{~h}$ interval, meaning that the computed shoreline contamination risk is dynamically responding to the variations of com- bined effects of metocean conditions and vessel traffic. The risks obtained at $\mathrm{P}_{1}$ (Praia Azul) during the simulation periods are in general larger than at $\mathrm{P}_{2}$, because the former point is located at a site subject to rougher metocean conditions. In winter, maximum risk values are identified on 19 and 23 January, in agreement with the peaks visible in winter metocean conditions, in Fig. 9. In summer, two strong peaks are identified at $\mathrm{P}_{1}$, on 21 and 23 January - both at 18:00 UTC. The latter one can be explained by the metocean conditions (significant wave height, wind and surface water velocity increase during that period, as can be seen in Fig. 10); however, the 


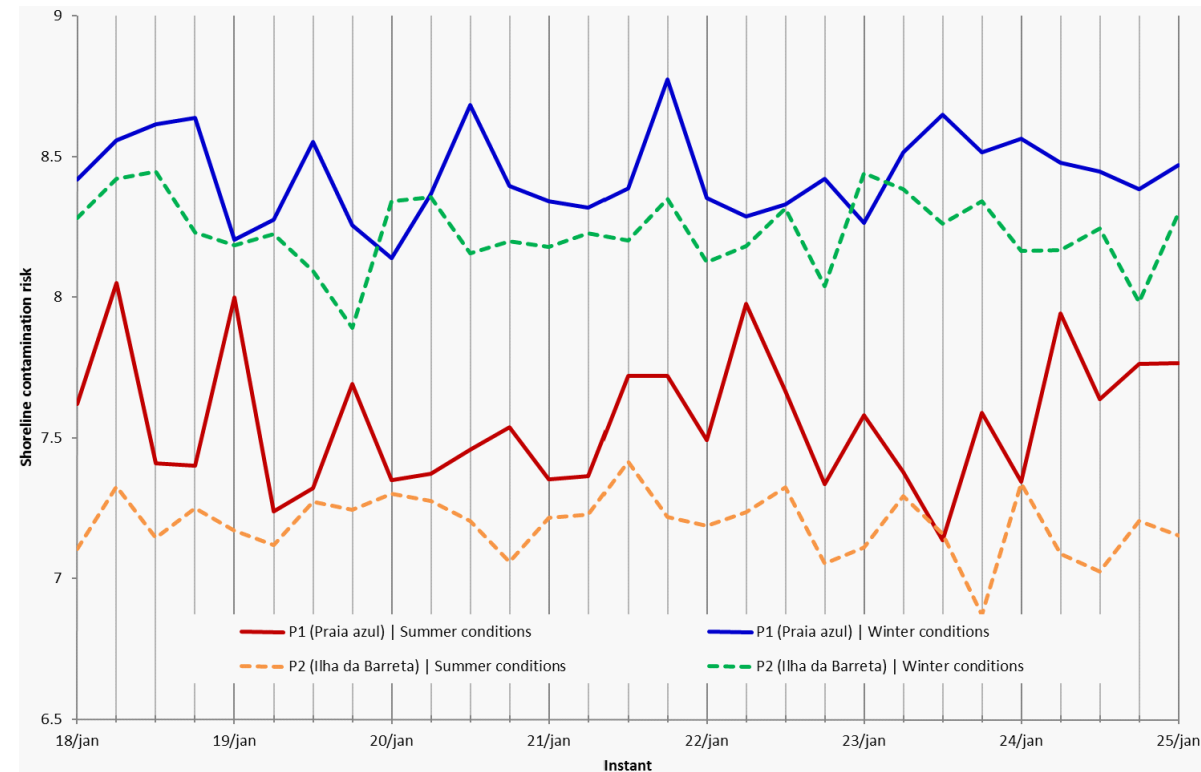

Figure 12. Integrated shoreline contamination risk for the whole pilot area, with AIS vessel information between 18 and 25 January 2013 , and using different space and time constant metocean conditions. Winter/rough conditions: surface current velocity, $0.55 \mathrm{~m} \mathrm{~s}^{-1}$; wind velocity, $15 \mathrm{~m} \mathrm{~s}^{-1}$; significant wave height, $3 \mathrm{~m}$. Summer/calm conditions: surface current velocity, $0.25 \mathrm{~m} \mathrm{~s}^{-1}$; wind velocity, $5 \mathrm{~m} \mathrm{~s}-1$; significant wave height, $1.5 \mathrm{~m}$.

former peak is only explained by the AIS information. Moreover, this peak is also present in summer (because the same AIS information is used).

\subsubsection{Integrated analysis in the whole pilot area}

Additionally to the evaluation of risk model behaviour in isolated shore locations, we performed a more complete and integrative set of analyses, considering all shore locations in the pilot area. In these analyses, the different shoreline contamination risks along the coast were integrated in the form of instant mean averages, and 1045 different shore locations along the Portuguese coast were considered. The risk model was run every 6 hours between 18 and 25 January 2013 (winter conditions), and between 18 and 25 June 2013 (summer conditions). The main purpose of performing these integrated analyses in the whole studied area is to obtain a more representative evaluation of the model risk behaviour.

The first integrated analysis for the whole area of study consisted in running the risk model using the same conditions as used on Sect. 3.3.1: constant metocean conditions in each period, in both space and time along the runs - thus with temporal evolution of the risk levels totally dependent on vessel traffic conditions. The summer scenario was run using vessel AIS information from the period between 18 and 25 June 2013: surface current velocity, $0.25 \mathrm{~m} \mathrm{~s}^{-1}$; wind velocity, $5 \mathrm{~m} \mathrm{~s}^{-1}$; significant wave height, $1.5 \mathrm{~m}$. The winter scenario was established with the vessel AIS information from 18 to 25 January 2013: surface current velocity, $0.55 \mathrm{~m} \mathrm{~s}^{-1}$; wind velocity, $15 \mathrm{~m} \mathrm{~s}^{-1}$; significant wave height,
$3 \mathrm{~m}$. The obtained mean and maximum values for the whole domain can vary more than 0.5 risk units at each time step $(6 \mathrm{~h})$, confirming the results obtained for $\mathrm{P}_{1}$ and $\mathrm{P}_{2}$. Obtained results from this analysis are presented in Fig. 12. The instant standard deviation values for the whole domain are usually around 0.5 for both runs. Most of the peaks detected in the risk mean and maximum values are around 12:00 and 18:00 $\mathrm{h}$ for both runs, meaning that this is the daily time period where most intense traffic seems to be detected in the studied area.

Additionally to the previously specified constant metocean conditions used, a set of three simulations was run for each of the selected periods (18-25 January; 18-25 June 2013), using the exact modelled metocean solutions provided by the operational forecasting systems, and additionally increasing and decreasing those solutions in $50 \%$ for the properties that directly affect the risk level. The main purpose of this set of simulations is to obtain a more sensitive analysis of the risk methodology under different realistic conditions, assuming that the chosen modelled scenarios will cover a representative part of the marine weather situations found in the pilot area. This set of analyses can also provide a clearer idea about the thresholds of the presented tool. The results from this set of simulations are shown in Fig. 13. These images also provide information about the maximum values and standard deviation for each instant, showing the dynamic variation along the coast.

The rougher metocean conditions previously identified for 19 January are responsible for a peak in shoreline contamination risk on that day, in winter conditions (Fig. 13a). In 


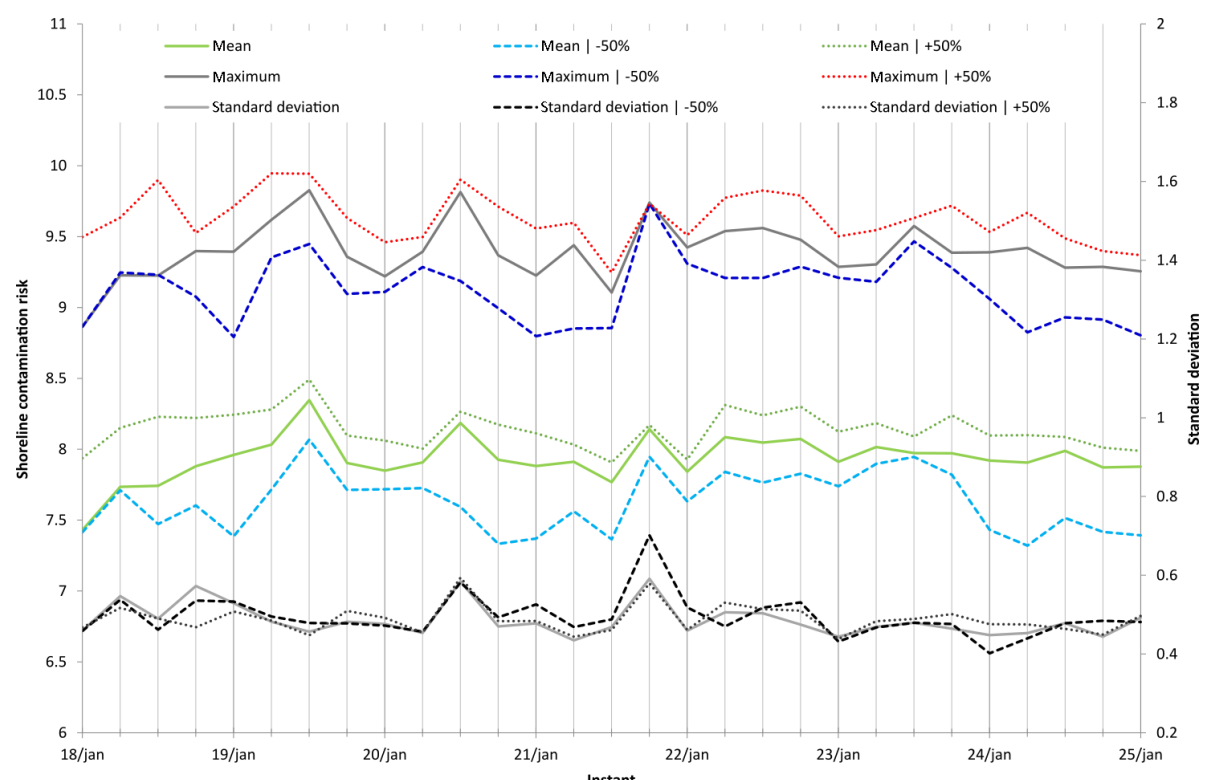

(a)

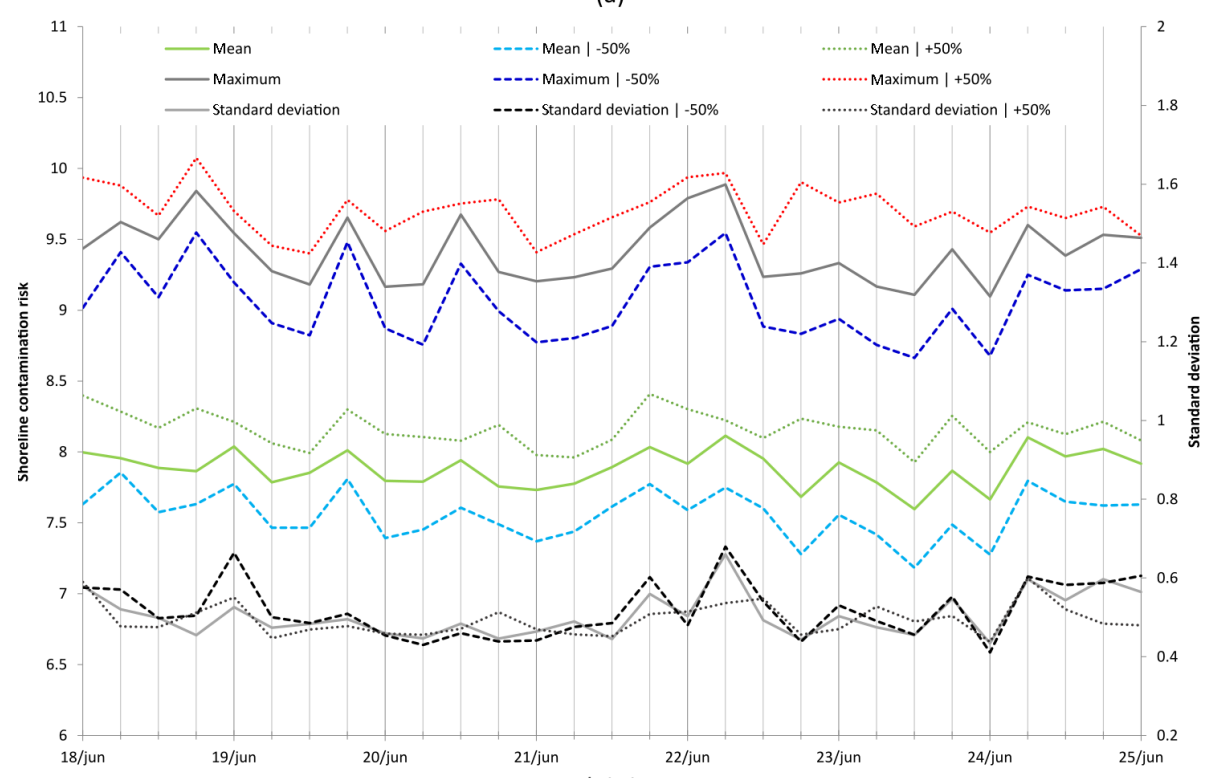

(b)

Figure 13. Integrated shoreline contamination risks (instant mean, maximum and standard deviation) for the whole pilot area, obtained with three values for metocean model parameters (direct model output; model $+50 \%$; model $-50 \%$ ). AIS vessel information and metocean model conditions between 18 and 25 January 2013 (a), and with AIS vessel information and metocean model conditions between 18 and 25 January 2013 (b).

each of Fig. 13a and b, the increase of metocean parameters generates an increase on computed risk levels, both mean and maximum values in the whole domain. Increasing or decreasing by 1.5 times $(50 \%)$ the metocean properties can result in a modification of risk levels up to 0.5 risk units. Mean risk values are generally around 8 risk units, with maximum risk values of 10, which is below the critical risk threshold (12) defined in the risk matrix (Table 2). Once again, instant standard deviations are generally around 0.5 or even higher, and the obtained mean and maximum values for the whole domain can vary more than 0.5 risk units at each time step $(6 \mathrm{~h})$, in both periods (although stronger variations are detected in January, which can also be explained by the stronger irregularity in metocean conditions for that period). These results confirm the previous analyses conducted for $\mathrm{P}_{1}$ and $\mathrm{P}_{2}$ and also for the whole domain, when using constant metocean conditions. 


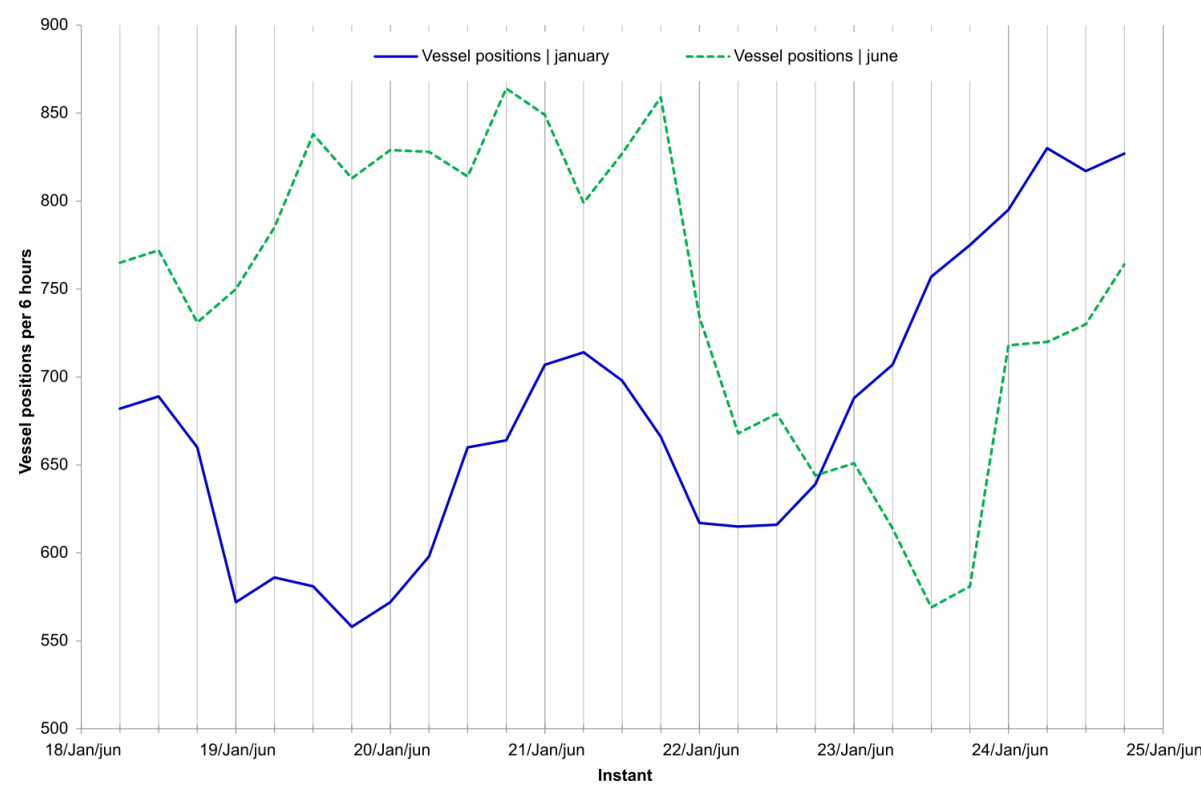

Figure 14. Recorded AIS vessel positions per $6 \mathrm{~h}$ time intervals in the pilot area, obtained in two different weeks: 18-25 January and 18-25 June 2013.

Comparing Fig. 13a and b, it can also be seen that the modelled risk is not necessarily lower in the summer period, despite the calmer metocean conditions. Indeed, in the first days of the simulations, the opposite situation is verified shoreline risk level is greater in summer. The main reason is the fact that vessel traffic was denser in the summer period, compared to the winter period (Fig. 14). The records indicate an average of 677 vessel positions recorded every $6 \mathrm{~h}$, during the selected January period. In the selected June period, an average of 725 vessel positions was recorded. Therefore, after comparing the integrated risk levels between Fig. 13a and $\mathrm{b}$, it can be said that in the modelled periods, the vessel traffic assumed more importance than the metocean conditions in the determination of the risk. Actually, the simple presence of a small number of mega-tankers in the nearshore is enough to increase the risk values. This also demonstrates the complexity of the system.

\subsection{The role of oil spill model}

The different metocean conditions directly affect accident probabilities (through correction factors), but can also influence oil weathering processes - for instance, higher evaporation rates are expected in the summer, reducing oil amount reaching shoreline, and consequently reducing shoreline risk in summer - as identified in Olita et al. (2012). On the other hand, stronger wind conditions in winter can also cause a more intense oil dispersion in the water column, contributing to a lower shoreline contamination risk in winter, as expressed in Liubartseva et al. (2015). The direct influence of oil weathering processes are studied in this chapter.
Different tests were performed to evaluate the relevance of having an oil fate and behaviour spill model integrated in this risk modelling tool. Calibration tests were also performed.

\subsubsection{Onshore vs. offshore}

First, it is important to evaluate the risk model response to different environmental conditions, favourable or unfavourable to shoreline spill contamination. In that sense, two opposite environmental modelling scenarios were defined in this scope, as the basis of the exercise here proposed: the same ship position and metocean conditions were used in both scenarios, except for wind direction (wind magnitude was not modified). The onshore wind scenario was set with a wind direction of $240^{\circ}$, favourable to transport oil to the near-shore. The offshore wind scenario was set with a wind direction of $60^{\circ}$, favourable to transport oil to the open ocean and far away from the coast. The risk model was then run for the whole pilot area for the two previously mentioned scenarios in different time instants during 1 day, and shoreline contamination risk levels for each time instant were integrated into mean values for the whole pilot area.

Since the developed risk model includes two different methods to compute the shoreline contamination risk (estimation of oil reaching the shoreline based on oil spill model - "modelled" approach; or based on ship proximity to shoreline - "non-modelled" approach), the two previous runs are also interesting to evaluate the relative dynamic response of the "modelled" shoreline contamination risk against the "non-modelled" approach, which therefore is independent of wind or current directions (thus there is no onshore and 


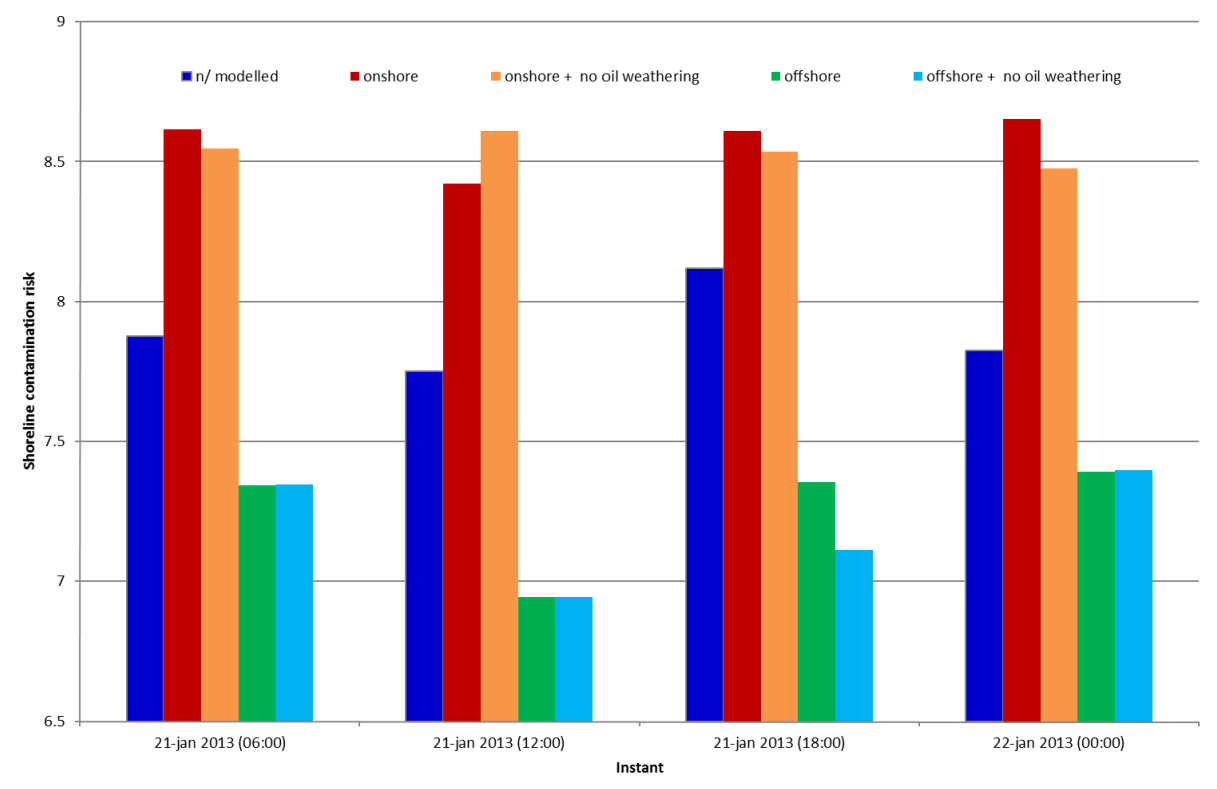

Figure 15. Integrated shoreline contamination risk levels at different time instants from 21 and 22 January 2013. Results presented as mean values for the shoreline in the whole pilot area studied. Shoreline risk levels computed with four different approaches: non-modelled approach; modelled approach using onshore wind; modelled approach without oil weathering processes, using onshore wind; modelled approach using offshore wind; modelled approach without oil weathering processes, using offshore wind.

offshore differentiation for the shoreline contamination risk computed using a "non-modelled" approach).

Additionally to the three previous runs, two more runs were included, turning off the oil spill weathering processes in both onshore and offshore wind scenarios. These two runs consisted in understanding how significant it is to integrate the oil-spill-specific weathering processes (mainly the oil spreading, evaporation, dispersion and emulsification) in the risk model, instead of simply using a generic Lagrangian model.

Those five different types of shoreline contamination results (non-modelled approach; on-shore wind scenario; offshore wind scenario; on-shore wind scenario with no oil weathering processes; offshore wind scenario with no oil weathering processes) were organized as mean values, see Fig. 15.

The results allow us to, firstly, understand the relevance of including an oil transport model in the risk approach, mainly because it reduces the predicted risk according to favourable metocean conditions (in this case, the wind direction) - the difference between on-shore wind scenario and the others is very significant. Secondly, it can be seen that the developed risk model benefits from the modelling of the oil weathering processes, as there is a difference between onshore wind scenario with and without oil weathering processes. The default oil product used (a medium crude oil named Carpinteria) has a relatively low evaporation rate (more significant in the first hours) and almost null dispersion. However, Carpinteria has significant emulsification potential, able to generate a polluted emulsion (with a high water content) with more mass than the initial oil spilt. In other words, this oil product, once spilt in water and subject to weathering processes, can increase its mass (through the incorporation of water in oil), therefore increasing the amount of pollutant reaching the shoreline, and increasing the risk of contamination when compared to shoreline contamination risk computed without oil weathering processes. This is in fact what is observed in some instants from Fig. 15.

\subsubsection{Comparing different oil products}

The adoption of Carpinteria as the default oil product for risk modelling is based on a "worst-case scenario" approach, related to the environmental problems that it can pose to shoreline areas due to low evaporation and dispersion, and significant emulsification. Using other oil products in this risk model would result in different risk values, due to differences in oil mass lost from the surface (to atmosphere, water column, etc.) related to oil weathering. To test the influence of different oil products in the risk model, a new set of tests with four different oil products was performed, using the previously defined environmental scenarios - onshore and offshore wind conditions.

The oil products selected have different weathering behaviour (Table 3).

Results for the onshore wind scenario are presented in Fig. 16 (results from the offshore wind scenario are not presented, since they show the same behaviour pattern as onshore conditions, although with lower risk values). Carpinteria keeps generating higher risk values, due to mass increase 
Table 3. Evolution in time of approximated oil mass lost and water content in oil (as percentage of mass) as a result of the main weathering processes, in four oil types, under regular metocean conditions in the pilot area (wind: $10 \mathrm{~m} \mathrm{~s}^{-1}$; significant wave height: $2.5 \mathrm{~m}$; water temperature: $\left.15^{\circ} \mathrm{C}\right)$.

\begin{tabular}{llrrr}
\hline \multirow{2}{*}{ Oil type } & Oil weathering process & \multicolumn{3}{c}{ Time after spill (h) } \\
\cline { 2 - 4 } & & 6 & 12 & 24 \\
\hline \multirow{2}{*}{ Diesel Fuel Oil (refined) } & Evaporation & $61 \%$ & $61 \%$ & $61 \%$ \\
& Dispersion & $39 \%$ & $39 \%$ & $39 \%$ \\
& Water content (emulsification) & - & - & - \\
\hline \multirow{2}{*}{ Carpinteria (crude) } & Evaporation & $33 \%$ & $34 \%$ & $36 \%$ \\
& Dispersion & $0.71 \%$ & $0.71 \%$ & $0.71 \%$ \\
& Water content (emulsification) & $70 \%$ & $72 \%$ & $72 \%$ \\
\hline \multirow{2}{*}{ Bunker C (refined) } & Evaporation & $4 \%$ & $5 \%$ & $6 \%$ \\
& Dispersion & $3 \%$ & $8 \%$ & $15 \%$ \\
& Water content (emulsification) & - & - & - \\
\hline \multirow{2}{*}{ Fuel Oil No 2 (refined) } & Evaporation & $13 \%$ & $14 \%$ & $14 \%$ \\
& Dispersion & $70 \%$ & $86 \%$ & $86 \%$ \\
& Water content in (emulsification) & - & - & - \\
\hline
\end{tabular}

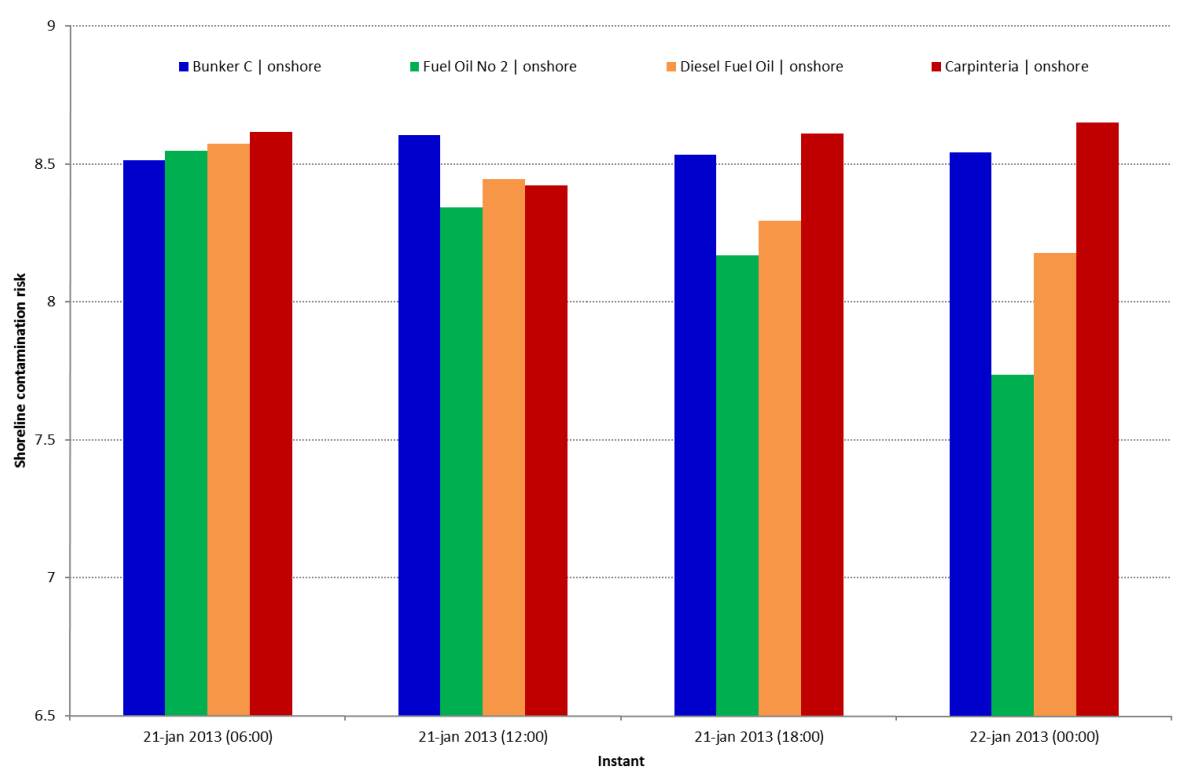

Figure 16. Integrated shoreline contamination risk levels at different time instants from 21 and 22 January 2013, obtained using four different oil products (Bunker C, Fuel Oil no. 2, Diesel Fuel Oil, Carpinteria), under onshore wind.

(emulsification). Bunker $\mathrm{C}$ fuel oil, which is a heavy fuel oil with low weathering effect, tends to generate risk values similar to Carpinteria. Diesel fuel oil and Fuel oil no. 2 tend to generate lower risk values, because they usually have more significant weathering processes - particularly diesel fuel oil.

\subsubsection{Calibration procedures}

A side test that was implemented during the development and implementation phase was the calibration of the risk model, specifically in the adoption of the parameter shoreline stretch extension unit - $L$ (Eq. 12). Increasing this value will increase the value obtained for the amount of oil reaching the coastline and the consequences in the risk model - and therefore increases the relative weight of oil spill model results in the risk model. Shoreline contamination risk levels were computed using $L=100 \mathrm{~m}$ and $L=1000 \mathrm{~m}$, for both onshore and offshore scenarios. Results presented in Fig. 17 show a constant increase of approximately 0.5 risk units when using $L=1000 \mathrm{~m}$ instead of $L=100 \mathrm{~m}$. Knowing that the mean values related to non-modelled risk of shoreline contamination risk, obtained in Sect. 3.3, are usually around 


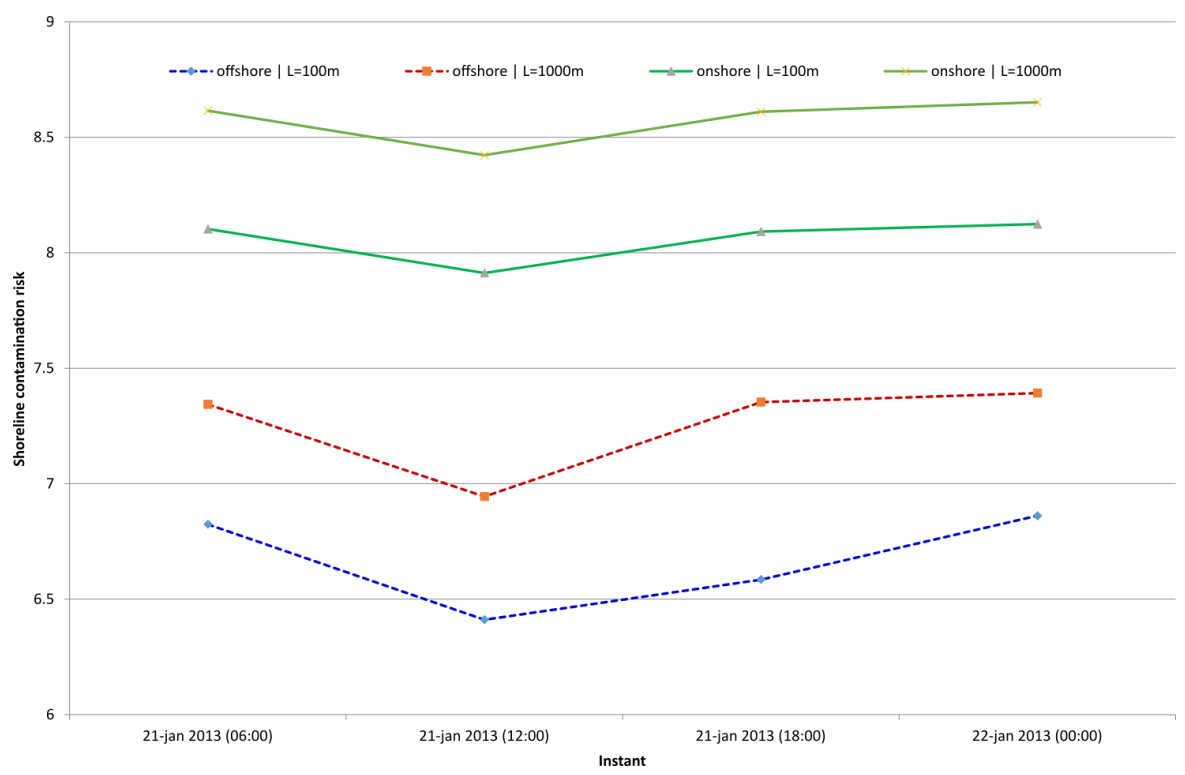

Figure 17. Integrated shoreline contamination risk levels at different time instants from 21 and 22 January 2013, under onshore and offshore wind, and using different values in parameter $L$ (shoreline stretch extension unit).

8 unit values, it is considered that $L=1000 \mathrm{~m}$ represents a reasonable approximation, as the value of 8 also fits the average values obtained by both exaggerated scenarios used (offshore and onshore wind conditions). Using $L=100 \mathrm{~m}$ generates lower risks, even in the onshore scenario. Adopting $L=100 \mathrm{~m}$ could therefore result in an underestimation of risk values, because we use the same risk scale as the one used for the non-modelled shoreline contamination risk.

\section{Discussion}

The work developed in this study aimed at the conceptualization, development and implementation of a novel holistic methodology for dynamic spill risk assessment from ship traffic, fully integrated with metocean and oil spill forecasting systems, and to evaluate the dynamic behaviour and response of the risk levels under different parameters. These objectives were accomplished, since the risk methodology was fully implemented in a software tool, the dynamic behaviour of the risk was demonstrated in the pilot area, and the system is being tested operationally by the authors of the project as well as the Portuguese Maritime Authority DGAM-SCPM, allowing it to be used both in real-time (providing support to monitoring activities) and on-demand situations (supporting contingency planning).

The software system here described has been designed to be easily transferable to other areas, adopting generic approaches to download specific data layers (e.g. metocean forecasting system, AIS data, etc.), and being easily usercustomized in terms of risk model parametrization. The possibility of running the risk model in a central server and pro- viding outputs to external platforms following OGC standards increases the interoperability of the system.

The role of different variables in the risk model was presented with specific examples, with special emphasis on the relative significance of metocean, vessel traffic conditions and oil spill modelling systems integrated for the pilot area. The results from the risk modelling software tool are in agreement with what was expected from the proposed methodology for risk. Using an oil transport model (together with metocean modelling systems) in the estimation of the risk of oil reaching the coastline can provide a more robust and dynamic risk assessment. The results presented here have shown that the mere fact of having intensive ship traffic in the proximity of some coastal areas does not necessarily mean that the risk of shoreline contamination is high, depending on the instantaneous metocean conditions. If they are favourable to transport an eventual oil spill to offshore, the risk of shoreline contamination will be low. Also, it was shown that even if the metocean and the sea state conditions are stable and not extremely rough (reducing the probability of having ship accidents) - the risk of having ship incidents may not be necessarily reduced, depending on a combination of multiple dynamic factors, including the ship traffic intensity.

The results obtained from the sensitive analysis to different metocean conditions suggested that the correction factors in terms of probability could eventually by intensified in the future, in order to increase the relative weight of metocean conditions in the risk model, and therefore the dynamic risk change based on marine weather conditions.

The inclusion of oil weathering processes in the determination of shoreline contamination risk generates differences in risk values, depending on the oil product considered in the 
risk model. By default, the risk model uses an oil product that represents a worst-case scenario for the shoreline (low evaporation and dispersion; high incorporation of water through emulsion). Calibration tests in the risk model were also pursued, in terms of consequences (e.g. increasing the relative weight of oil spill model results in the risk model), in order to improve and fine tune the performance.

In all the results presented in this work (including conditions very favourable to shoreline oil pollution, calm and rough metocean conditions), the mean and maximum risk values tend to be below the critical risk threshold defined in the risk matrix presented in Table 2 (critical risk values are between 12 and 16). Therefore, the predefined risk matrix may be adapted in the future to better reflect the minimum and maximum values detected in the pilot area. In parallel, less relative weight to the coastal vulnerability indices might be used, in order to increase the amplitude and dynamic component of the risk, associated with vessel traffic, metocean conditions and the oil spill weathering model.

Additionally, it should be noted that this study was mainly focused on the testing and evaluation of the risk model dynamic behaviour and response to the different variables, and comparing the amplitude of risk values in the pilot area. The evolution of risk values over longer time periods was beyond the scope of the study at this stage. This type of study is expected to be pursued in the future, for the same pilot area included in this work.

Independently of the methodology developed and the results achieved with this study, a number of assumptions, limitations and lack of data were identified as relevant for improving the risk model:

- Using frequency constants to estimate the probability of having incidents may need continuous and periodic update, because the frequent changes in the ship industry (e.g. obligation of double hull ships, mega-tankers, maritime surveillance, etc.) can change the probability of having incidents.

- The coastal vulnerability indices included should also be regularly updated and reviewed to reflect the present situation in terms of environment and socio-economic aspects of the coast.

- Several schemes have been developed for estimating the probability of ship-to-ship collisions using more complex approaches (e.g. Silveira et al., 2013); however, these algorithms have not been included yet in this risk model.

- Heterogeneous spatial resolutions were considered for the different variables used in the risk model. We have assumed that the computed risk index resolution is equal to the coastal vulnerability (which has a high resolution $-200 \mathrm{~m}$ or less - allowing responders to properly visualize, manage and prioritize different shoreline areas), but we have in mind that a better spatial resolution of the metocean models would potentially improve representation of the coastal processes and consequently, the risk model. The software tool is ready to accommodate more metocean models with higher spatial resolution, which can be particularly interesting when studying or monitoring the risk levels at a local scale (e.g. in a Port and its neighbourhood area). Nevertheless, as a first dynamic implementation and for the regional purpose of this work (focused on the Portuguese/Western Iberian shelf), we consider that the proposed approach is capable of demonstrating and providing satisfactory results. Moreover, the included metocean models have been previously reported as valid for studying coastal processes and coastal management support (Mateus et al., 2012; Trancoso, 2012; Franz et al., 2014)

- In the risk model adopted, there is no differentiation between identical ships from different countries, inspected at different ports, constructed or managed by different companies, or with different number of deficiencies detected in the recent past. This information is presently available online through EMSA's THETIS system, and in the future can be seen as a relevant added value for integration in the risk model, if possible.

- The actual volume of contaminants, and product type transported by each ship is not included in the risk model, since the information is not publicly available (an approximation based on ship type and dead weight tonnage is adopted). This information would be rather important to improve the realistic quantification of estimated risk.

- No risk acceptance or tolerability criteria were defined in the present risk model. The future definition of these tolerability criteria will facilitate the adoption of mitigation measures in the case of unacceptable/intolerable risks detected.

Aside from these identified considerations, the work presented here opens interesting opportunities for the future both in terms of risk planning and monitoring activities. A tool like this can improve the decision support model, allowing the prioritization of individual ships or geographical areas, and facilitating strategic and dynamic tug positioning. The possibility of being used for past or hypothetical scenarios may provide an interesting tool not only for identifying "hotspots" in terms of shoreline contamination risk, but also to estimate future situations like the increasing of ship traffic or the size and cargo transported by the ships. Furthermore, the same risk model approach can be considered in the future to estimate other types of environmental threats, including impacts from spills in offshore platforms, impacts from onshore activities and industries involving discharges to the water environment, or even the environmental impact of maritime transport emissions on coastal air quality. 


\section{Appendix A: Coastal vulnerability indices}

This section provides additional detail about the classification adopted for the coastal vulnerability indices adopted in the pilot area, namely the coastal sensitivity index (CSI, Table A1) and the socio-economic index (SESI, Table A2).

Table A1. Classes used for coastal sensitivity index (CSI).

\begin{tabular}{|c|c|c|c|c|c|}
\hline \multirow[t]{2}{*}{ Colour } & \multirow[t]{2}{*}{ CSI } & \multicolumn{3}{|c|}{ Colour code (RGB) } & \multirow[t]{2}{*}{ CSI and type of shoreline } \\
\hline & & $\mathrm{R}$ & G & $\mathrm{B}$ & \\
\hline & 1 & 119 & 38 & 105 & $\begin{array}{l}\text { 1A: exposed rocky shores } \\
\text { 1B: Exposed, solid man-made structures }\end{array}$ \\
\hline & 2 & 174 & 153 & 191 & Exposed wave-cut platform in bedrock, mud, or clay. Medium slope \\
\hline & 3 & 0 & 151 & 212 & Exposed fine- to medium-grained sand dissipative beaches \\
\hline & 4 & 146 & 209 & 241 & $\begin{array}{l}\text { Exposed beaches with coarse-grained or fine- to medium-grained sand; } \\
\text { sheltered beaches with fine-grained sand }\end{array}$ \\
\hline & 5 & 152 & 206 & 201 & Mixed sand and gravel beaches \\
\hline & 6 & 0 & 149 & 32 & $\begin{array}{l}\text { 6A: Gravel beaches } \\
\text { 6B: Riprap }\end{array}$ \\
\hline & 7 & 214 & 186 & 0 & Exposed tidal flats \\
\hline & 8 & 225 & 232 & 0 & $\begin{array}{l}\text { 8A: Sheltered scarps in bedrock, mud or clay } \\
\text { 8B: Sheltered, solid man-made structures }\end{array}$ \\
\hline & 9 & 248 & 163 & 0 & $\begin{array}{l}\text { 9A: Sheltered tidal flats } \\
\text { 9B: Sheltered low banks }\end{array}$ \\
\hline & 10 & 214 & 0 & 24 & $\begin{array}{l}\text { Salt and brackish waters marsh, freshwater marshes, } \\
\text { swamps, mangroves or scrub wetlands }\end{array}$ \\
\hline
\end{tabular}


Table A2. Classes used for socio-economical index (SESI).

\begin{tabular}{|c|c|}
\hline SESI & Description \\
\hline 1 & $\begin{array}{l}\text { Area of none or very low importance in terms of environmental resources, leisure and other sea-related activities. } \\
\text { Specific interests of the area are affected by the spill. The human population does not live directly or indirectly on the } \\
\text { resources provided by sea-related activities. }\end{array}$ \\
\hline 2 & $\begin{array}{l}\text { Area of low importance in terms of environmental resources, leisure and other sea-related activities; area of local interest. } \\
\text { There is low investment that may be affected by the spill; some interests of the area are affected by the spill. }\end{array}$ \\
\hline 3 & $\begin{array}{l}\text { Area of medium importance in terms of environmental resources, leisure and other sea-related activities; } \\
\text { area of medium regional and national interest. } \\
\text { There is medium investment that may be affected by the spill. The spill affects the economy of the area and few } \\
\text { economic aspects of neighbouring areas. }\end{array}$ \\
\hline 4 & $\begin{array}{l}\text { Area of high importance in terms of environmental resources, leisure and other sea-related activities; } \\
\text { area of high regional and national interest. } \\
\text { The human population lives directly or indirectly on the resources provided by sea-related activities. The economy of the area } \\
\text { and neighbouring areas can be affected by the spill; or there is high investment that may be affected by the spill. }\end{array}$ \\
\hline 5 & $\begin{array}{l}\text { Area of extreme importance in terms of environmental resources, leisure and other sea-related activities; } \\
\text { area of very high regional and national interest. } \\
\text { There is very high investment and economy of the area that may be affected by the spill. } \\
\text { The human population lives directly or indirectly from the resources provided by sea-related activities. }\end{array}$ \\
\hline
\end{tabular}


Table B1. Different types of risk of spill incidents, and corresponding spill incident frequency constants.

\begin{tabular}{|c|c|c|c|}
\hline Navigation & Type of incident & Risk index & $\begin{array}{l}\text { Accident Frequency } \\
\text { per km navigated }\end{array}$ \\
\hline $\begin{array}{l}\text { Ship navigating in } \\
\text { restricted waters }\end{array}$ & $\begin{array}{l}\text { Ship-to-ship collision } \\
\text { Collision with port facilities } \\
\text { Grounding } \\
\text { Fire and explosion }\end{array}$ & 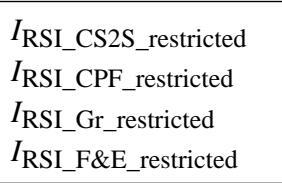 & $\begin{array}{l}3.52 \times 10^{-7 \mathrm{a}} \\
4.22 \times 10^{-7 \mathrm{~b}} \\
2.83 \times 10^{-7} \mathrm{~b} \\
1.78 \times 10^{-7 \mathrm{a}}\end{array}$ \\
\hline $\begin{array}{l}\text { Ship navigating in } \\
\text { unrestricted waters }\end{array}$ & $\begin{array}{l}\text { Ship-to-ship collision } \\
\text { Foundering and structural failures } \\
\text { Grounding during navigation } \\
\text { Drift grounding } \\
\text { Fire and explosion } \\
\text { Illegal/Operational Discharges }\end{array}$ & $\begin{array}{l}I_{\text {RSI_CS2S_unrestricted }} \\
I_{\text {RSI_Fo_unrestricted }} \\
I_{\text {RSI_GDN_unrestricted }} \\
I_{\text {RSI_DG_unrestricted }} \\
I_{\text {RSI_F\&E_unrestricted }} \\
I_{\text {RSI_IOD_unrestricted }}\end{array}$ & $\begin{array}{l}1.26 \times 10^{-8 \mathrm{a}} \\
9.17 \times 10^{-8 \mathrm{~b}} \\
1.23 \times 10^{-7} \mathrm{~b} \\
1.89 \times 10^{-8 \mathrm{~b}} \\
1.78 \times 10^{-7 \mathrm{a}} \\
2.49 \times 10^{-5} \mathrm{~b} \text { (annual frequency) }\end{array}$ \\
\hline
\end{tabular}

\section{Appendix B: Background on risk of spill incident}

Table B1 describes the types of incidents considered in the risk model, as well as the nomenclature used and the corresponding spill incident frequency constants (per distance unit navigated or annual frequency for illegal/operational discharges).

In the determination of these risk indices for each type of incident, the generic risk formula (sum of probability and severity indices) applies. For example, for ships navigating in restricted waters, the risk of spill incident from a ship-toship collision is

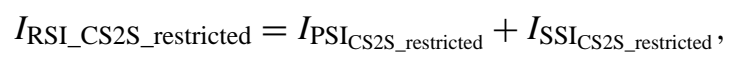

where $I_{\mathrm{PSI}_{\mathrm{CS} 2 \mathrm{~S} \text { restricted }}}$ and $I_{\mathrm{SSI} \_\mathrm{CS} 2 \mathrm{~S} \_ \text {restricted }}$ are the probability index and severity index (respectively) for ship-to-ship collision in restricted waters.

An integrated risk index is also determined $\left(I_{\mathrm{RSI}}\right)$, which means that we can also estimate the risk of an incident of a specific ship, independently of the type of incident. This integrated risk index is a sum of the various probability indices $\left(I_{\sum \text { PSI_restricted }}\right)$ with the weighted arithmetic mean of the severity indices $\left(\overline{I_{\text {SSI_restricted }}}\right)$ from the different types of incidents.

Thus, if a ship is navigating in restricted waters:

$I_{\text {IRSI_restricted }}=I_{\sum \mathrm{P}_{\text {rrestricted }}}+\overline{I_{\text {SSI_restricted }}}$,

where $I_{\sum}$ PSI_restricted is computed as follows:

$$
\begin{aligned}
I_{\sum \text { PSI_restricted }}= & f\left(P_{\mathrm{CS} 2 \mathrm{~S} \_ \text {restricted }}+P_{\mathrm{CPF} \_ \text {restricted }}\right. \\
& \left.+P_{\mathrm{Gr} \_ \text {restricted }}+P_{\mathrm{F} \& \mathrm{E}_{\text {_restricted }}}\right),
\end{aligned}
$$

where $P_{\mathrm{CS} 2 \mathrm{~S} \text { _restricted }}$ is the probability of ship-to-ship collision in restricted waters, $P_{\mathrm{CPF} \text { _restricted }}$ is the probability of collision to port facilities in restricted waters, $P_{\text {Gr_restricted }}$ is the probability of grounding in restricted waters, $P_{\mathrm{F} \& \mathrm{E} \_ \text {restricted }}$ is the probability of fire and explosion in restricted waters, and $\overline{I_{\text {SSI_restricted }}}$ is computed as follows:

$\overline{I_{\text {SSI_restricted }}}=$

$\frac{\left(P_{\text {CS2S_restricted }} \times I_{\text {SSI }} \text { CS2S_restricted }\right)+\left(P_{\text {CPF_restricted }} \times I_{\text {SSI }_{\text {CPF_restricted }}}\right)}{\sum P_{\text {restricted }}}$ $+\frac{\left(P_{\mathrm{Gr} \_ \text {restricted }} \times I_{\mathrm{SSI}} \mathrm{Gr} \_ \text {restricted }\right)+\left(P_{\mathrm{FEE} \_ \text {restricted }} \times I_{\mathrm{SSI}_{\mathrm{F \& E} \_ \text {restricted }}}\right)}{\sum P_{\text {restricted }}}$,

where $\sum P_{\text {restricted }}$ means the sum of probabilities in restricted waters.

Alternatively, if a ship is navigating in unrestricted waters, a similar approach is followed:

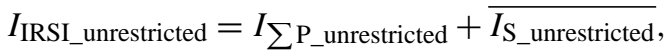

where $I_{\sum}$ PSI_unrestricted and $\overline{I_{\text {SSI_restricted }}}$ mean the sum of probability indices and the weighted arithmetic mean of severity indices, both in unrestricted waters. $I_{\sum \text { PSI_unrestricted }}$ is computed as follows:

$$
\begin{aligned}
& I_{\sum} \mathrm{P} \_ \text {unrestricted } \\
& f\left(P_{\mathrm{CS} 2 \mathrm{~S} \_ \text {unrestricted }}+P_{\mathrm{Fo} \_ \text {unrestricted }}+P_{\mathrm{GDN} \_ \text {unrestricted }}\right. \\
& \left.+P_{\mathrm{DG} \_ \text {unrestricted }}+P_{\mathrm{F} \& \mathrm{E} \_ \text {unrestricted }}+P_{\mathrm{IOD} \_ \text {unrestricted }}\right),
\end{aligned}
$$

where $P_{\mathrm{CS} 2 \mathrm{~S} \text { _unrestricted }}$ is the probability of ship-to-ship collision in unrestricted waters, $P_{\text {Fo_unrestricted }}$ is the probability of foundering and structural failures in unrestricted waters, $P_{\mathrm{GDN} \_u n r e s t r i c t e d}$ is the probability of grounding during navigation in unrestricted waters, $P_{\mathrm{DG} \_ \text {runestricted }}$ is the probability of drift grounding, $P_{\mathrm{F} \& \mathrm{E} \_ \text {unrestricted }}$ is the probability of 
fire and explosion in unrestricted waters, and $P_{\text {IOD_unrestricted }}$ is the probability of illegal/operational discharge in unrestricted waters. $I_{\text {SSI unrestricted }}$ is computed as follows:

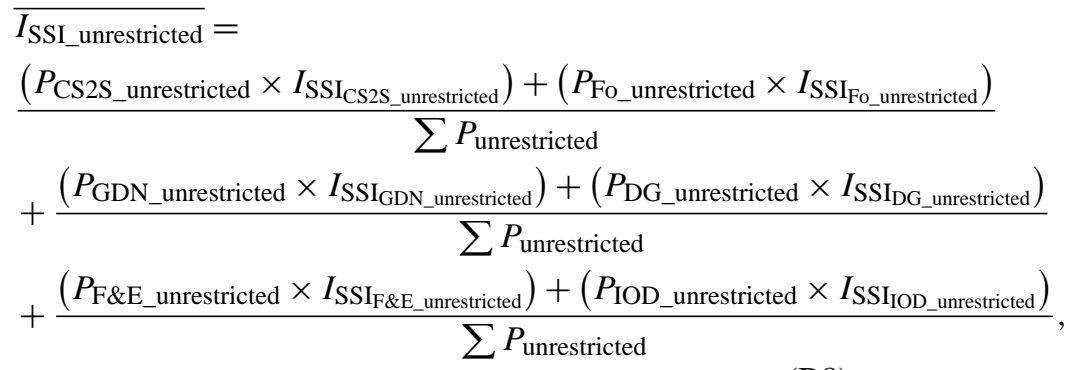

where $\sum P_{\text {unrestricted }}$ means the sum of probabilities in unrestricted waters.

\section{Appendix C: Background on probability estimation}

To estimate the index of probability (Table $\mathrm{C} 1$ ), frequency constants obtained from reported spill incidents are used (per distance unit navigated or annual frequency for illegal/operational discharges) for the various types of accidents. Frequency constant values are shown in Table B1.

The probability of spill incidents is influenced by certain conditions that can reduce or increase the probability. The developed risk model includes correction factors to take into consideration these conditions. Table $\mathrm{C} 2$ expresses the correction factors adopted.

Table C1. Classification of probability of ship incidents and correspondence between annual probability and index of probability (obtained from Filipe and Pratas, 2007, and inspired by IMO recommendation - IMO, 2002)

\begin{tabular}{llll}
\hline $\begin{array}{l}\text { Probability/ } \\
\text { frequency }\end{array}$ & Definition & $\begin{array}{l}\text { Annual probability/ } \\
\text { frequency }\left(P_{\text {annual }}\right)\end{array}$ & $\begin{array}{l}\text { Index of } \\
\text { probability }\left(I_{\mathrm{PSI}}\right)\end{array}$ \\
\hline Very high & Likely to occur once or more a month & $10-100$ or more & $>7-8$ \\
High & Likely to occur once to 10 times a year & $1-10$ & $>6-7$ \\
Medium & Likely to occur once in a period from 1 to 10 years & $10^{-2}-1$ & $>4-6$ \\
Low & Likely to occur from 0.5 to $50 \%$ within a period of 50 years & $10^{-4}-10^{-2}$ & $>2-4$ \\
Very low & Likely to occur from 0.05 to $0.5 \%$ within a period of 50 years & $10^{-5}-10^{-4}$ & $0-2$ \\
\hline
\end{tabular}




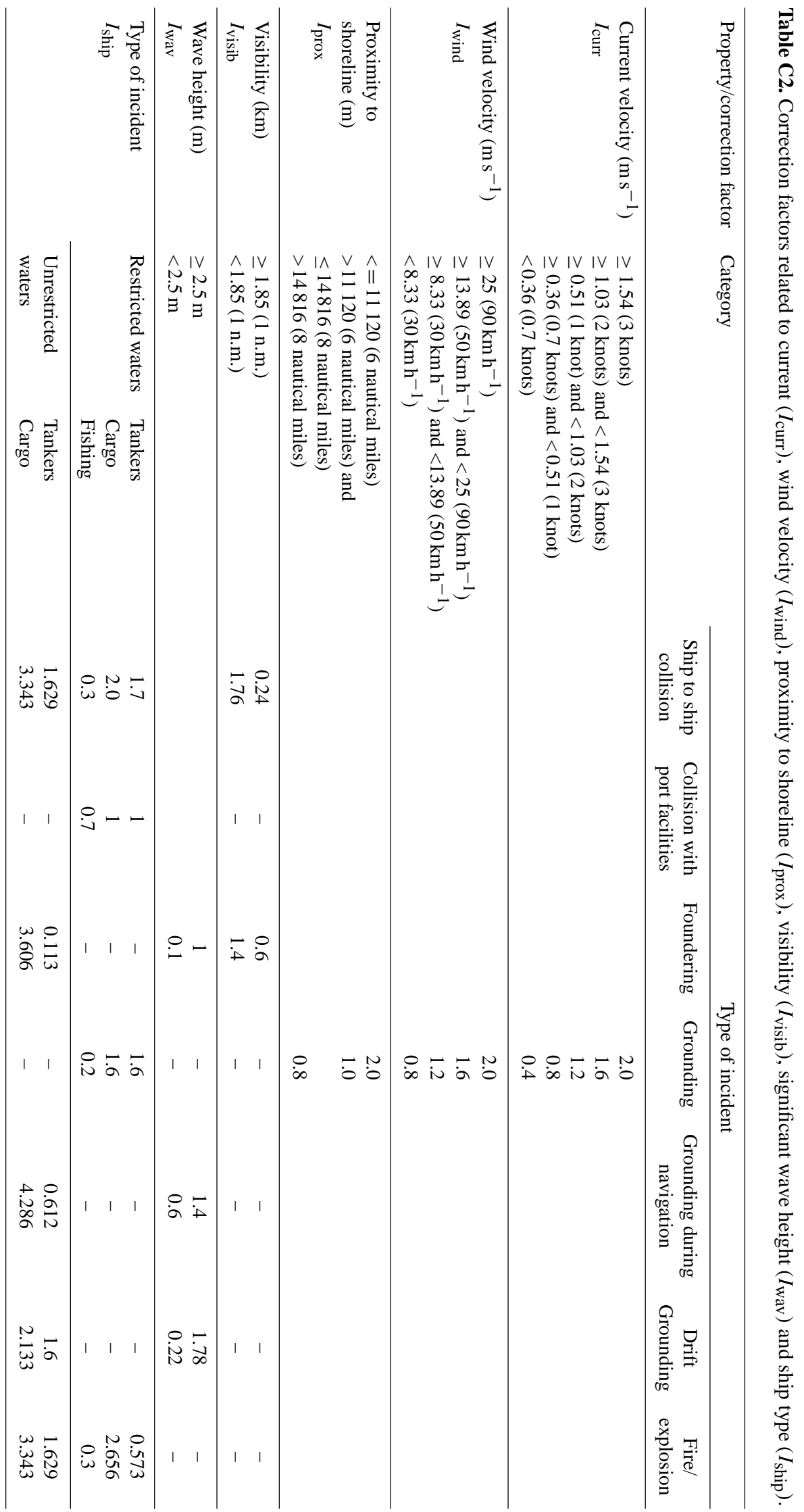




\section{Appendix D: Background on severity estimation}

Table D1 shows the correspondence between severity/consequences and index of severity, obtained from Filipe and Pratas (2007) and inspired by IMO recommendation (IMO, 2002).

Table D2 illustrates how to determine the amount of oil spilled $(Q)$ based on dead weight (DW) and ship type, and Table D3 expresses the methods for determination of severity indices based on the oil amount computed in Table D2. The equations from Tables D2 and D3 were obtained from Filipe and Pratas (2007).
The computation of severity of non-modelled risk of shoreline contamination includes the subtraction of a correction factor $\left(F_{\mathrm{SS}}\right)$ that depends on distance between spill site and shoreline $\left(D_{\mathrm{SS}}\right)$, and on type of oil product/ship type, as expressed in Table D4. The values used here are based on Filipe and Pratas (2007). In that report the correction factor was applied on a scale between 1 and 15 , and in this work the correction factor is applied in the severity index between 1 and 8 , so a multiplying factor of $8 / 15$ is applied to transform the correction factor to the appropriate scale.

Table D1. Classification of severity of ship incidents and correspondence between severity and index of severity.

\begin{tabular}{|c|c|c|c|c|}
\hline \multirow{2}{*}{$\begin{array}{l}\text { Severity } \\
\text { degree }\end{array}$} & \multicolumn{3}{|c|}{ Impacts } & \multirow{2}{*}{$\begin{array}{l}\text { Severity } \\
\text { index } \\
\left(I_{\mathrm{SSI}}\right)\end{array}$} \\
\hline & Human health & Environment & $\begin{array}{l}\text { Socio-economical activi- } \\
\text { ties }\end{array}$ & \\
\hline Catastrophic & $\begin{array}{l}\text { Catastrophic number of } \\
\text { injuries, fatalities and } \\
\text { physical disabilities }\end{array}$ & $\begin{array}{l}\text { Catastrophic and permanent damage to } \\
\text { the marine flora and fauna }\end{array}$ & $\begin{array}{l}\text { Affecting at a catastrophic } \\
\text { scale and for long periods } \\
\text { of time }\end{array}$ & $>7-8$ \\
\hline Extreme & $\begin{array}{l}\text { Extremely number of } \\
\text { injuries, fatalities and } \\
\text { physical disabilities }\end{array}$ & $\begin{array}{l}\text { Extreme and permanent damage to the } \\
\text { marine flora and fauna }\end{array}$ & $\begin{array}{l}\text { Affecting at extreme scale } \\
\text { and for long periods of } \\
\text { time }\end{array}$ & $>6-7$ \\
\hline $\begin{array}{l}\text { Very high or } \\
\text { very serious }\end{array}$ & $\begin{array}{l}\text { Very high number of } \\
\text { injuries, fatalities and } \\
\text { physical disabilities }\end{array}$ & $\begin{array}{l}\text { Very serious and almost permanent } \\
\text { damage to the marine flora and fauna }\end{array}$ & $\begin{array}{l}\text { Affecting at very high } \\
\text { scale and for long periods } \\
\text { of time }\end{array}$ & $>5-6$ \\
\hline $\begin{array}{l}\text { High or } \\
\text { serious }\end{array}$ & $\begin{array}{l}\text { High number of } \\
\text { injuries, or physical } \\
\text { disabilities }\end{array}$ & $\begin{array}{l}\text { Long-term damage to the marine flora and } \\
\text { fauna; high cost of measures needed to re- } \\
\text { store the resources affected by the spill }\end{array}$ & $\begin{array}{l}\text { Affecting at high scale and } \\
\text { for long periods of time }\end{array}$ & $>4-5$ \\
\hline $\begin{array}{l}\text { Medium or } \\
\text { moderate }\end{array}$ & $\begin{array}{l}\text { Medium number of } \\
\text { injuries (unlikely to } \\
\text { result in physical } \\
\text { disabilities) }\end{array}$ & $\begin{array}{l}\text { Medium-term damage to the marine flora } \\
\text { and fauna; moderate cost of measures } \\
\text { needed to restore the resources affected by } \\
\text { the spill }\end{array}$ & $\begin{array}{l}\text { Affecting at medium scale } \\
\text { and for long periods of } \\
\text { time }\end{array}$ & $>3-4$ \\
\hline $\begin{array}{l}\text { Little or } \\
\text { slight }\end{array}$ & Few injuries & $\begin{array}{l}\text { Short-term damage to the marine flora and } \\
\text { fauna; low cost of measures needed to } \\
\text { restore the resources affected by the spill }\end{array}$ & $\begin{array}{l}\text { Affecting at small scale } \\
\text { and for long periods of } \\
\text { time }\end{array}$ & $>2-3$ \\
\hline $\begin{array}{l}\text { Very little } \\
\text { or very } \\
\text { slight }\end{array}$ & $\begin{array}{l}\text { Very few injuries; very } \\
\text { little first aid assistance }\end{array}$ & $\begin{array}{l}\text { Very short term damage to the marine } \\
\text { flora and fauna; very low cost of measures } \\
\text { needed to restore the resources affected by } \\
\text { the spill }\end{array}$ & $\begin{array}{l}\text { Affecting at small scale } \\
\text { and for long periods of } \\
\text { time }\end{array}$ & $>1-2$ \\
\hline Insignificant & $\begin{array}{l}\text { No reported harm to } \\
\text { human health }\end{array}$ & $\begin{array}{l}\text { No damage to the marine flora and fauna. } \\
\text { No restoration measures needed }\end{array}$ & No effects & $>0-1$ \\
\hline
\end{tabular}


Table D2. Average amount of spilled oil per incident type and ship type.

\begin{tabular}{llll}
\hline Type of incident & Tanker (crude) & Fishing vessels (diesel) & Cargo (bunker) \\
\hline Ship to ship collision & $Q=1 \times 10^{-7} \mathrm{DW}^{2}+0.0327 \mathrm{DW}$ & $Q=6$ & $Q=60$ \\
Collision with port facilities & $Q=5 \times 10^{-8} \mathrm{DW}^{2}+0.0134 \mathrm{DW}$ & $Q=3$ & $Q=25$ \\
Foundering & $Q=\mathrm{DW}$ & $Q=12$ & $Q=1300$ \\
Grounding & $Q=5 \times 10^{-7} \mathrm{DW}^{2}+0.1362 \mathrm{DW}$ & $Q=2$ & $Q=130$ \\
Fire and explosion & $Q=0.8 \mathrm{DW}$ & $Q=10$ & $Q=100$ \\
Illegal/operational discharges & $Q=25^{*}$ & $Q=3^{*}$ & $Q=7^{*}$ \\
\hline
\end{tabular}

$Q=$ oil amount (ton); DW $=$ Deadweight (DWT). ${ }^{*}$ Values used are the worst case values/highest values for the different types of operational/illegal discharges.

Table D3. Quantification of severity index of spill incident, based on oil amount ship type.

\begin{tabular}{lll}
\hline Ship type & Unrestricted waters & Restricted waters \\
\hline Crude (tanker) & $I_{\text {SSI_unsrestricted }}=0.4037 \ln (Q)+1.9534$ & $I_{\text {SSI_restricted }}=0.4693 \ln (Q)+1.9903$ \\
& $I_{\text {SSI_unrestrictedmin }}=0 ; I_{\text {SSI_unrestricted_max }}=8$ & $I_{\text {SSI_unrestricted_min }}=0 ; I_{\text {SSI_restricted_max }}=8$ \\
\hline \multirow{2}{*}{ Diesel (fishing) } & $I_{\text {SSI_unrestricted }}=0.4343 \ln (Q)+1.301$ & $I_{\text {SSI_restricted }}=0.4689 \ln (Q)+1.666$ \\
& $I_{\text {SSI_restrictedmin }}=0 ; I_{\text {SSI_unrestricted_max }}=7$ & $I_{\text {SSI_restrictedmin }}=0 ; I_{\text {SSI_restrictedmax }}=8$ \\
\hline \multirow{2}{*}{ Bunker (cargo) } & $I_{\text {SSI }}=0.3996 \ln (Q)+1.9285$ & $I_{\text {SSI_restricted }}=0.4517 \ln (Q)+2.1643$ \\
& $I_{\text {SSI_restrictedmin }}=0 ; I_{\text {SSI_unrestricted_max }}=8$ & $I_{\text {SSI_restrictedmin }}=0 ; I_{\text {SSI_restrictedmax }}=8$ \\
\hline
\end{tabular}

Table D4. Subtracting correction factor $\left(F_{\mathrm{SS}}\right)$ based on spill site used, in function of ship type and distance between spill site and shoreline $\left(D_{\mathrm{SS}}\right)$.

\begin{tabular}{ll}
\hline Ship Type & Equation for correction factor $\left(F_{\mathrm{SS}}\right)$ \\
\hline Fishing (Diesel) & $F_{\mathrm{SS}}=\frac{8}{15} \cdot 0.3 \cdot D_{\mathrm{SS}}$ \\
Tanker (Crude) & $F_{\mathrm{SS}}=\frac{8}{15} \cdot 0.2 \cdot D_{\mathrm{SS}}$ \\
Cargo (Bunker) & $F_{\mathrm{SS}}=\frac{8}{15} \cdot 0.1 \cdot D_{\mathrm{SS}}$ \\
\hline
\end{tabular}


Acknowledgements. This work has been sponsored by projects ARCOPOL PLUS (2011-1/150) and ARCOPOL PLATFORM (20131/252) (EU Atlantic Area).

The authors thank Francisco Campuzano for the development and maintenance of the hydrodynamic and wave modelling systems, as well as Rosa Trancoso for the development of atmospheric model, and Jorge Palma for its operational maintenance. The authors want also to thank MarineTraffic for cooperation and the provision of AIS data support in the scope of the ARCOPOL PLUS project. Last, but not least, a special thanks to the Portuguese Maritime Authority (DGAM-SCPM) for cooperation and support in beta testing.

Edited by: A. Crise

\section{References}

Ascione Kenov, I., Campuzano, F., Franz, G., Fernandes, R., Viegas, C., Sobrinho, J., de Pablo, H., Amaral, A., Pinto, L., Mateus, M., and Neves, R.: Advances in modeling of water quality in estuaries, in: Remote Sensing and Modeling, edited by: Finkl, C. W., and Makowski, C., Springer International Publishing, 237-276, doi:10.1007/978-3-319-06326-3_10, 2014.

Bi, H. and Si, H.: Dynamic risk assessment of oil spill scenario for Three Gorges Reservoir in China based on numerical simulation, Safety Sci., 50, 1112-1118, doi:10.1016/j.ssci.2011.11.012, 2012.

Canu, D., Solidoro, C., Bandelj, V., Quattrocchi, G., Sorgente, R., Olita, A., Fazioli, L., and Cucco, A.: Assessment of oil slick hazard and risk at vulnerable coastal sites, Mar. Pollut. Bull., 94, 84-95, doi:10.1016/j.marpolbul.2015.03.006, 2015.

Carracedo, P., Torres-López, S., Barreiro, M., Montero, P., Balseiro, C. F., Penabad, E., Leitão, P. C., and Pérez-Munuzuri, V.: Improvement of pollutant drift forecast system applied to the prestige oil spills in Galicia Coast (NW of Spain): development of an operational system, Mar. Pollut. Bull., 53, 350-360, doi:10.1016/j.marpolbul.2005.11.014, 2006.

Castanedo, S., Abascal, A. J., Medina, R., Fernandez, F., Liste, M., and Olabarrieta, M.: Development of a GIS-based oil spill risk assessment system, OCEANS 2009 - EUROPE, Bremen, 1-6, doi:10.1109/OCEANSE.2009.5278283, 2009.

den Boer, S., Azevedo, A., Vaz, L., Costa, R., Fortunato, A. B., Oliveira, A., Tomás, L. M., Dias, J. M., and Rodrigues, M.: Development of an oil spill hazard scenarios database for risk assessment, in: Proceedings 13th International Coastal Symposium, Durban, South Africa, edited by: Green, A. N., and Cooper, J. A. G., J. Coastal Res., 70, 539-544, 2014.

DGPM (Directorate-General for Political Sea): Economics of the Sea in Portugal (document to support the National Ocean Strategy 2013-2020), available at: http://www.dgpm.mam.gov.pt/ Documents/Anexo_A.pdf, 2012.

Eide, M. S., Endresen, Ø., Breivik, Ø., Brude, O. W., Ellingsen, I. H., Røang, K., Hauge, J., and Brett, P. O.: Prevention of oil spill from shipping by modelling of dynamic risk, Mar. Pollut. Bull., 54, 1619-1633, doi:10.1016/j.marpolbul.2007.06.013, 2007a.

Eide, M. S., Endresen, Ø., Brett, P. O., Ervik, J. L., and Røang, K.: Intelligent ship traffic monitoring for oil spill prevention: risk based decision support building on AIS, Mar. Pollut. Bull., 54, 145-148, doi:10.1016/j.marpolbul.2006.11.004, 2007 b.

Etkin, D.: Chapter 1 - Risk analysis and prevention, in: Handbook of Oil Spill Science and Technology, edited by: Fingas, M., John Wiley \& Sons, Inc, Hoboken, NJ, doi:10.1002/9781118989982.ch1, 2014.

Fernandes, R.: Modelação de Derrames de Hidrocarbonetos, (in English: Modelling of Oil Spills) - Final Report in Environmental Engineering Degree, Instituto Superior Técnico, Lisboa, available at: http://www.mohid.com/PublicData/Products/ Thesis/TFC_RodrigoFernandes.pdf, 2001.

Fernandes, R., Neves, R., Viegas, C., and Leitão, P.: Integration of an oil and inert spill model in a framework for risk management of spills at sea: a case study for the Atlantic area, in: Proceedings of the Thirty-sixth AMOP Technical Seminar on Environmental Contamination and Response, Environment Canada, Ottawa, ON, 326-353, doi:10.13140/2.1.1740.3200, 2013.

Fernandes, R., Braunschweig, F., and Lourenço, F.: Dynamic Risk Analysis Apapted To Different Regional Needs: Dynamic Risk Tool Manual and Implementation Methodology, ARCOPOL PLUS Report, Instituto Superior Técnico, available at: http://www.arcopol.eu/?/=/section/resources/search/1/ resource/105, 2014.

Filipe, D. and Pratas, E.: Methodology for Risk Assessment of Accidents That Originate Hydrocarbon and Other Noxious and Hazardous Substances Spills at Sea, and Their Potential Impact, EROCIPS Project Emergency Response to Coastal Oil, Chemical and Inner Pollution from Shipping Interreg IIIB: Atlantic Area Programme, 2007.

Fingas, M.: Chapter 10 - Introduction to spill modeling, in: Handbook of Oil Spill Science and Technology, edited by: Fingas, M., John Wiley \& Sons, Inc, 287-299, doi:10.1002/9781118989982.ch4, 2015.

Fischer, Y. and Bauer, A.: Object-oriented sensor data fusion for wide maritime surveillance, in: Proceedings of 2 nd NURC International Waterside Security Conference, IEEE, 1-6, doi:10.1109/WSSC.2010.5730244, 2010.

Franz, G., Campuzano, F., Pinto, L., Fernandes, R., Sobrinho, J., Simões, A., Juliano, M., and Neves, R.: Implementation and validation of an operational wave modelling forecasting system for the Portuguese Coast, 7th EUROGOOS Conference, Lisbon, Portugal, 2014.

GESAMP: Joint Group of Experts on the Scientific Aspects of Marine Environmental Protection (GESAMP) - Report No. 75 "Estimates of Oil Entering the Marine Environment from Sea-Based Activities", 61, available at: http://www.gesamp.org/data/gesamp/files/media/Publications/ Reports_and_studies_75/gallery_1042/object_1042_large.pdf, 2007.

Goldman, R., Biton, E., Brokovich, E., Kark, S., and Levin, N.: Oil spill contamination probability in the southeastern Levantine basin, Mar. Pollut. Bull., 91, 347-356, doi:10.1016/j.marpolbul.2014.10.050, 2014.

Gong, Y., Zhao, X., Cai, Z., O'Reilly, S. E., Hao, X., and Zhao, D.: A review of oil, dispersed oil and sediment interactions in the aquatic environment: influence on the fate, transport and remediation of oil spills, Mar. Pollut. Bull., 79, 16-33, doi:10.1016/j.marpolbul.2013.12.024, 2014. 
Grell, G., Dudhia, J., and Stauffer, D.: A Description of the FifthGeneration Penn State/NCAR Mesoscale Model (MM5), available at: http://nldr.library.ucar.edu/repository/assets/technotes/ TECH-NOTE-000-000-000-214.pdf, 1994.

Grifoll, M., Jordà, G., Borja, Á., and Espino, M.: A new risk assessment method for water quality degradation in harbour domains, using hydrodynamic models, Mar. Pollut. Bull., 60, 6978, doi:10.1016/j.marpolbul.2009.08.030, 2010.

Hurlburt, H. E., Brassington, G. B., Drillet, Y., Kamachi, M., Benkiran, M., Bourdallé- Badie, R., Chassignet, E. P., Jacobs, G. A., Le Galloudec, O., Lellouche, J.-M., Metzger, E. J., Oke, P. R., Pugh, T. F., Schiller, A., Smedstad, O. M., Tranchant, B., Tsujino, H., Usui, N., and Wallcraft, A. J.: High resolution global and basin-scale ocean analyses and forecasts, Oceanography, 22, 110-127, doi:10.5670/oceanog.2009.70, 2009.

IAEA - International Atomic Energy Agency: Severity, Probability and Risk of Accidents During Maritime Transport of Radioactive Material, IAEA-TECDOC-1231, Vienna, available at: http://www-pub.iaea.org/MTCD/publications/PDF/te_1231_ prn.pdf, 2001.

IMO - International Maritime Organization: Guidelines for formal safety assessment (FSA) for use in the IMO rulemaking process, MSC/Circ.1023/MEPC/Circ.392, available at: http://www.imo.org/en/OurWork/HumanElement/ VisionPrinciplesGoals/Documents/1023-MEPC392.pdf, 2002.

ITOPF: Oil Tanker Statistics 2014, International Tanker Owners Pollution Federation Limited, available at: http://www.itopf.com/fileadmin/data/Documents/Company_ Lit/Oil_Spill_Stats_2014FINALlowres.pdf, 2005.

Janeiro, J., Zacharioudaki, A., Sarhadi, E., Neves, A., and Martins, F.: Enhancing the management response to oil spills in the Tuscany Archipelago through operational modelling, Mar. Pollut. Bull., 85, 574-589, doi:10.1016/j.marpolbul.2014.03.021, 2014.

Johansen, Ø., Reed, M., and Bodsberg, N. R.: Natural dispersion revisited, Mar. Pollut. Bull., 93, 20-26. doi:10.1016/j.marpolbul.2015.02.026, 2015.

Leitão, P. C., Malhadas, M., Ribeiro, J., Leitão, J., Pierini, J., and Otero, L.: An overview for simulating the blow out of oil spills with a three-dimensional model approach (Caribbean Coast, Colombia), in: Ocean Modelling for Coastal Management - Case Studies with MOHID, edited by: Mateus, M. and Neves, R., IST PRESS, 97-115, available at: http://www.mohid.com/books/ 2013OceanModellingMOHID.pdf, 2013.

Leschine, T. M.: Oil spills and the social amplification and attenuation of risk, Spill Sci. Technol. B., 7, 63-73, doi:10.1016/S13532561(02)00050-6, 2002.

Liubartseva, S., De Dominicis, M., Oddo, P., Coppini, G., Pinardi, N., Greggio, N.: Oil spill hazard from dispersal of oil along shipping lanes in the Southern Adriatic and Northern Ionian Seas, Mar. Pollut. Bull., 90, 259-272, doi:10.1016/j.marpolbul.2014.10.039, 2015.

MARETEC: Portuguese Coastal Atlas, available at: http://arcopol.maretec.org/CoastalAtlas/AtlasCosteiro_ PORTUGALCONTINENTAL_Netlink.kmz, 2009.

Mateus, M., Riflet, G., Chambel, P., Fernandes, L., Fernandes, R., Juliano, M., Campuzano, F., de Pablo, H., and Neves, R.: An operational model for the West Iberian coast: products and services, Ocean Sci., 8, 713-732, doi:10.5194/os-8-713-2012, 2012.
Neves, R.: The Mohid concept, in: Ocean Modelling for Coastal Management - Case Studies with MOHID, edited by: Mateus, M. and Neves, R., IST Press, 1-11, available at: http://www.mohid.com/PublicData/Products/BookPapers/ 2013_mohidbook_C01.pdf, 2013.

NOAA - National Oceanic and Atmospheric Administration: Environmental Sensitivity inde Guidelines - version 3.0, Seattle, NOAA Technical Memorandum NOS OR\&R 11, available at: http://response.restoration.noaa.gov/sites/default/files/ ESI_Guidelines.pdf, 2002.

Olita, A., Cucco, A., Simeone, S., Ribotti, A., Fazioli, L., Sorgente, B., and Sorgente, R.: Oil spill hazard and risk assessment for the shorelines of a Mediterranean coastal Archipelago, Ocean Coast. Manage., 57, 44-52, doi:10.1016/j.ocecoaman.2011.11.006, 2012.

Otero, P., Ruiz-Villarreal, M., Allen-Perkins, S., Vila, B., and Cabanas, J. M.: Coastal exposure to oil spill impacts from the Finisterre Traffic Separation Scheme, Mar. Pollut. Bull., 85, 67-77, doi:10.1016/j.marpolbul.2014.06.020, 2014.

Pinto, L., Campuzano, F., Fernandes, R., Fernandes, L., and Neves, R.: An operational model for the Portuguese coast, 2.as Jornadas de Engenharia Hidrográfica, Lisbon, 85-88, 2012.

Schiller, A.: Ocean forecasting in the 21 st century - from the early days to tomorrow's challenges, in: Operational Oceanography in the 21 st Century, edited by: Schiller, A. and Brassington, G., 3 26, doi:10.1007/978-94-007-0332-2_1, 2011.

Silveira, P. A. M., Teixeira, A. P., and Guedes Soares, C.: Use of AIS data to characterise marine traffic patterns and ship collision risk off the coast of Portugal, J. Navigation, 66, 879-898, doi:10.1017/S0373463313000519, 2013.

Sousa, T.: Previsão meteorológica em Portugal Continental utilizando um modelo operacional e de investigação MM5, M.Sc. thesis for the Msc. degree in environmental engineering, Instituto Superior Técnico, Universidade Técnica de Lisboa, Portugal, in Portuguese, available at: http://meteo.ist.utl.pt/public/ publicacoes/teses/Msc_TaniaSousa.pdf, 2002.

Tolman, H. L.: User Manual and System Documentation of WAVEWATCH III version 3.14 - Technical note, MMAB Contribution 276, available at: polar.ncep.noaa.gov/mmab/papers/tn276/ MMAB_276.pdf, 2009.

Tournadre, J.: Anthropogenic pressure on the open ocean: the growth of ship traffic revealed by altimeter data analysis, Geophys. Res. Lett., 41, 7924-7932, doi:10.1002/2014GL061786, 2014.

Trancoso, A. R.: Operational Modelling as a Tool in Wind Power Forecast and Meteorological Warnings, $\mathrm{PhD}$ thesis, Instituto Superior Técnico, Universidade Técnica de Lisboa, available at: http://meteo.ist.utl.pt/public/publicacoes/teses/ 2012_ARTrancoso_PhDThesis.pdf, 2012.

WSP Canada Inc.: Risk Assessment of Marine Spills in Canadian Waters, Phase 1: Oil Spills South of the 60th Parallel, Report Number: WSP 131-17593-00, Prepared for Transport Canada, available at: http://wcel.org/sites/default/files/file-downloads/ 131-17593-00_ERA_Oil-Spill-South_150116_pp1-124.pdf, 2014.

Zhao, L., Boufadel, M. C., Socolofsky, S. A., Adams, E., King, T., and Lee, K.: Evolution of droplets in subsea oil and gas blowouts: development and validation of the nu- 
merical model VDROP-J, Mar. Pollut. Bull., 83, 58-69. doi:10.1016/j.marpolbul.2014.04.020, 2014a.

Zhao, L., Torlapati, J., Boufadel, M. C., King, T., Robinson, B., and Lee, K.: VDROP: a comprehensive model for droplet formation of oils and gases in liquids - incorporation of the interfacial tension and droplet viscosity, Chem. Eng. J., 253, 93-106, doi:10.1016/j.cej.2014.04.082, 2014b. 